\title{
Generalized Levi Conditions for Weakly Hyperbolic Equations -An Attempt to Treat the Degeneracy with Respect to the Space Variables-
}

By

Takeshi MANDAI*

\section{$\S 0$. Introduction}

In the celebrated paper [3], Ivrii and Petkov showed that the effective hyperbolicity of an operator is necessary for the Cauchy problem to be $C^{\infty}$-wellposed with arbitrary lower order terms. In the same paper, they also gave general necessary conditions on lower order terms for the $C^{\infty}$-well-posedness. (Conditions of this kind are sometimes called "generalized Levi conditions".) The individual behavior of each characteristic root, however, is not reflected in these conditions. Hence, when some characteristic roots coincide with one another in a variety of ways, their results fail to give the conditions expected to be necessary and sufficient.

In [8], we treated each characteristic root separately, restricting our consideration to the degeneracy with respect to the time variable. We gave some necessary conditions for $C^{\infty}$-well-posedness and some estimates for the regularityloss of solutions. These results were "micro-local" in the sense that conditions were stated in terms of the characteristic root 0 in a fixed cotangential direction. By applying these results after appropriate coordinate transformations, however, we got a necessary and sufficient condition in case of the operators with only finite-order degeneracy w.r.t. the time variable ([9]).

This paper is an attempt to treat the degeneracy with respect to the space variables in a similar way. Though the results in this paper hold for the operators with singular coefficients of the same kind as discussed in [8] and [9], we restrict ourselves to the case of operators with $C^{\infty}$-coefficients for simplicity.

We introduce some notations for further explanation.

Communicated by S. Matsuura, March 20, 1985.

* Department of Mathematics, Faculty of General Education, Gifu University, Yanagido, Gifu, Japan. 


$$
\begin{aligned}
& (t, x)=\left(t, x_{1}, \cdots, x_{n}\right)=\left(t, x^{\prime}, x_{n}\right) \in \boldsymbol{R}^{n+1} \text {, } \\
& (\tau, \xi)=\left(\tau, \xi_{1}, \cdots, \xi_{n}\right)=\left(\tau, \xi^{\prime}, \xi_{n}\right) \in \boldsymbol{R}^{n+1} \text {. } \\
& \partial_{t}=\partial / \partial t, \quad \partial_{x_{j}}=\partial / \partial x_{j}, \quad D_{t}=-i \partial_{t}, \quad D_{x_{j}}=-i \partial_{x_{j}}, \\
& D_{x}^{\alpha}=D_{x_{1}}^{\alpha_{1}} \cdots D_{x_{n}}^{\alpha} \text {, where } \alpha=\left(\alpha_{1}, \cdots, \alpha_{n}\right) \text { is a multi-index. } \\
& q_{(k, \beta)}^{(j, \alpha)}(t, x ; \tau, \xi)=\left(\partial_{t}^{k} \partial_{x}^{\beta} \partial_{\tau}^{j} \partial_{\xi}^{\alpha} q\right)(t, x ; \tau, \xi) \text {, } \\
& \dot{\boldsymbol{R}}^{n}=\boldsymbol{R}^{n} \backslash\{0\}, \quad e_{n}=(0, \cdots, 0,1) \in \boldsymbol{R}^{n} .
\end{aligned}
$$

Let $\Omega$ be an open neighborhood of $(0,0) \in \boldsymbol{R}^{n+1}$ and let $\Omega^{+}=\{(t, x) \in \Omega ; t \geqq 0\}$. Let $P$ be a differential operator on $\Omega$ with $C^{\infty}$-coefficients and set $P=$ $\sum_{h=0}^{m} p_{m-h}\left(t, x ; D_{t}, D_{x}\right)$, where $p_{m-h}(t, x ; \tau, \xi)$ is homogeneous of degree $m-h$ with respect to $(\tau, \xi)$ and $p_{m}(t, x ; 1,0)=1$ on $\Omega$.

To avoid ambiguity, the definition of well-posedness will be specified as follows.

Definition 0.1. We say that the Cauchy problem for $P$ is well-posed on $\Omega^{+}$ if the following two conditions are satisfied.

a) For any $f \in C^{\infty}(\Omega)$ such that $f=0$ for $t \leqq 0$, there exists $u \in C^{\infty}(\Omega)$ such that $u=0$ for $t \leqq 0$ and $P u=f$ on $\Omega$.

b) For any $\hat{t} \geqq 0$ and any $u \in C^{\infty}(\Omega)$ such that $u=0$ for $t \leqq 0$, if there holds $P u=0$ for $t \leqq \hat{t}$, then there holds $u=0$ for $t \leqq \hat{t}$.

Remark 0.2 . 1) In [3], [8] etc., certain conditions on dependence domains are assumed. In this paper, we do not make such assumptions. Further, the results in this paper hold under some other definitions of well-posedness. (See Remark 1.3 1).)

2) If the coefficients of $P$ are $C^{\infty}$ as we have assumed, then this definition is equivalent to the following condition $\mathrm{c}$ ).

c) For any $\hat{t} \geqq 0$, any $f \in C^{\infty}(\Omega)$ and any $g_{j} \in C^{\infty}(\Omega \cap\{t=\hat{t}\})(j=0,1, \cdots$, $m-1)$, there exists a unique $u \in C^{\infty}(\Omega)$ such that $P u=f$ for $t \geqq \hat{t}$ and $\partial_{t}^{j} u_{1 t=\hat{t}}=g_{j}$ $(j=0,1, \cdots, m-1)$.

Since the well-posedness implies hyperbolicity ([10]), we assume throughout this paper that $P$ is hyperbolic on $\Omega^{+}$, that is, the equation $p_{m}(t, x ; \tau, \xi)=0$ has only real roots $\tau$ for any $(t, x ; \xi) \in \Omega^{+} \times \boldsymbol{R}^{n}$.

Now, we review the simplest version of the "Levi condition" ([7], [11], [12], [2]). Let $p_{m-h}\left(t, x ; \tau, e_{n}\right)=a_{0}^{(h)}(t, x) \tau^{m-h}+\cdots+a_{m-h}^{(h)}(t, x)$. If $a_{m}^{(0)}(t, x)=\cdots=$ $a_{m-r+1}^{(0)}(t, x)=0$ on $\Omega^{+}$and the Cauchy problem for $P$ is well-posed on $\Omega^{+}$, then there holds $a_{m-h}^{(h)}(t, x)=\cdots=a_{m-r+1}^{(h)}(t, x)=0$ on $\Omega^{+}$for $h=1, \cdots, r-1$. This is the condition to be called the "micro-local Levi condition with respect to the characteristic root 0 in $e_{n}$-direction". If the characteristic roots of $P$ have 
constant multiplicities, then the usual Levi condition, which is also a sufficient condition, is obtained by combining the conditions which are obtained as above after transforming $P$ by appropriate coordinate transformations. In this paper, we are concerned with the case where $a_{m}^{(0)}(t, x), \cdots, a_{m-r+1}^{(0)}(t, x)$ vanish at $(0,0)$ but do not vanish identically near $(0,0)$. To describe the behavior of $a_{j}^{(0)}(t, x)$ $(j=0, \cdots, m)$ near $(0,0)$, the Newton polygon shall play an important role.

Definition 0.3. We call a subset $\Delta$ of $\boldsymbol{R}^{2}$ a Newton polygon of size $m$ ( $m$ is a non-negative integer) if $\Delta=\{(j, \mu) \in[0, m] \times \mathbb{R} ; \mu \geqq \Gamma(j)\}$, where $\Gamma(j)$ has one of the following forms.
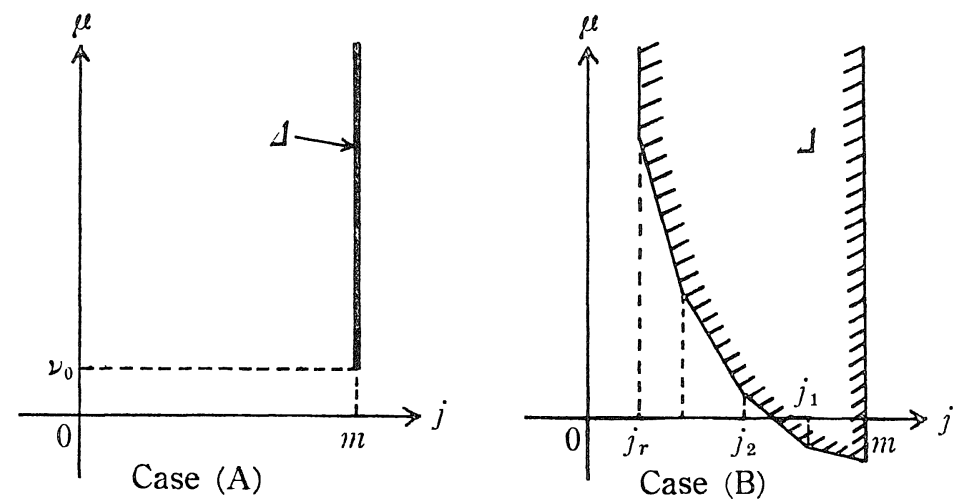

Figure 1.

(A) $\Gamma(m)=\nu_{0}$ and $\Gamma(j)=\infty$ for $0 \leqq j<m$, where $\nu_{0}$ is a rational number.

(B) $\Gamma(j)=\nu_{k}-\kappa_{k} j$ for $j_{k} \leqq j \leqq j_{k-1}(k=1, \cdots, r)$ and $\Gamma(j)=\infty$ for $0 \leqq j<j_{r}$, where $r$ is a positive integer, $m=j_{0}>j_{1}>\cdots>j_{r} \geqq 0$ are integers and $\nu_{1}<\nu_{2}<\cdots<\nu_{r}, 0 \leqq \kappa_{1}<\kappa_{2}<\cdots<\kappa_{r}$ are rational numbers.

The function $\mu=\Gamma(j)(0 \leqq j \leqq m)$ is called the lower side of $\Delta$.

In $\S 1$, we shall give necessary conditions for well-posedness with respect to the characteristic root 0 (Theorem 1.2). This theorem is an extension of Theorems 4.1, 7.1 in [3]. The proof is given in $\S 3$. In $\S 2$, we shall consider a class of " $t$-involutive" operators (Definition 2.1). For such operators, a necessary and sufficient condition is established (Theorem 2.5). The proof is given in $\S 4$ by applying Theorem 1.2 after appropriate coordinate transformations.

\section{$\S 1$. Generalized Levi Conditions with Respect to the Characteristic Root 0}

Fix positive rational numbers $q_{j}(j=0,1, \cdots, n)$. Put $\mathcal{L}=\left\{\left(j, q_{0} k+\langle q, \beta\rangle\right)\right.$ $\left.\dot{\in} \mathbb{R}^{2} ; p_{m,(k, \beta)}^{(j, 0)}\left(0,0 ; 0, e_{n}\right) \neq 0\right\}$, where $\langle q, \beta\rangle=\sum_{j=1}^{n} q_{j} \beta_{j}$. Let $\Delta$ be the closed convex 


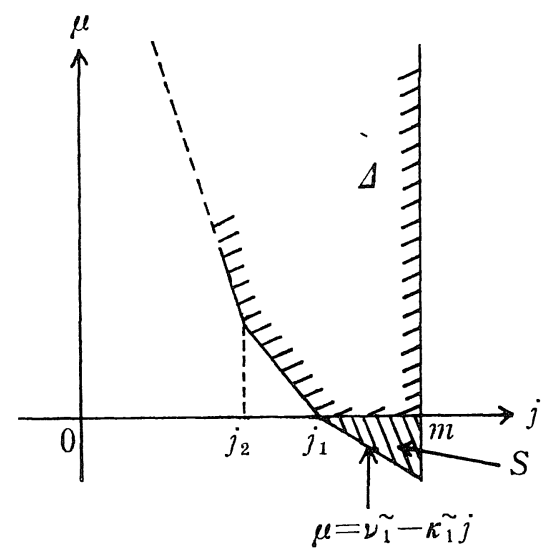

Figure 2

hull of the set $\left\{(j, \mu) \in \mathbb{R}^{2} ; \mu \geqq \mu^{\prime}\right.$ for some $\left.\left(j, \mu^{\prime}\right) \in \mathscr{Q}\right\}$ in $\boldsymbol{R}^{2}$. This is a Newton polygon. Since the coefficients are $C^{\infty}$ and $p_{m}(t, x ; 1,0)=1$, there holds $(m, 0)$ $\in \Delta \subset\left\{(j, \mu) \in R^{2} ; \mu \geqq 0\right\}$. For this $\Delta$, we get $\nu_{0}$ (case (A)) or $r, j k, \kappa_{k}$ and $\nu_{k}$ $(1 \leqq k \leqq r)$ (case $(\mathrm{B}))$ as in Definition 0.3. In the case (B) with $\kappa_{1}=0$ (hence $\left.\nu_{1}=0\right)$, we may modify $\Delta$ as follows. Take an arbitrary rational number $\tilde{\kappa_{1}}$ such that $0 \leqq \tilde{\kappa_{1}} \leqq \kappa_{2}$ and put $\tilde{\nu_{1}}=\tilde{\kappa_{1}} j_{1}$. Let $S$ be the closed triangle with the vertexes $(m, 0),\left(j_{1}, 0\right),\left(m, \tilde{\nu}-\tilde{\kappa_{1}} m\right)$ and let $\Delta^{\sim}=\Delta \cup S$. This is another Newton polygon (Figure 2). In the case (B) with $\kappa_{1}>0$, we make no modification, that is, put $\tilde{\kappa_{1}}=\kappa_{1}$ and $\tilde{\Delta}^{\sim}=\Delta$. In the case $(\mathrm{A})$, put $\tilde{\kappa_{1}}=\infty$ and $\tilde{\Delta}^{\sim}=\Delta$. We make the following assumptions on $q_{j}(0 \leqq j \leqq n), \tilde{\kappa_{1}}$ and $p_{m}$. In other words, we consider only $q_{3}, \tilde{\kappa_{1}}$ that satisfy the following conditions.

[A] $\begin{cases}1) & q_{0}+\tilde{\kappa_{1}}>q_{j} \\ 2) & \text { If }\left(j+|\alpha|, q_{0}(k+|\alpha|)+\langle q, \beta-\alpha\rangle\right) \notin \Delta^{\sim}, \text { then } p_{m,(k, \beta)}^{(j, a)}\left(0,0 ; 0, e_{n}\right)=0 .\end{cases}$

Remark 1.1. i) For $\alpha=0$, the condition 2) is satisfied by the definition of $\Delta^{\sim}$. ii) If $q_{0} \geqq q_{j}(j=1,2, \cdots, n)$, then the condition 2$)$ follows from the hyperbolicity of $p_{m}$. (The proof is given in Appendix 1.)

iii) Assume 1). Then, the condition 2) for such $\alpha$ as satisfy $\alpha_{n}=0$ implies the condition 2) for all $\alpha$. Especially, the condition 2) is necessarily satisfied if $n=1$.

Theorem 1.2. Assume the condition [A]. If the Cauchy problem for $P$ is well-posed on $\Omega^{+}$, then the following holds:

If $\left(j+|\alpha|+h, q_{0}(k+|\alpha|+h)+\langle q, \beta-\alpha\rangle\right) \notin \Delta^{\sim}$, then $p_{m-h,(k, \beta)}^{(j, \alpha)}\left(0,0 ; 0, e_{n}\right)=0$.

Remark 1.3. 1) The assumption of well-posedness is used only to derive the energy inequality (3-3) in $\S 3$. Hence, any definition of well-posedness that 
implies this inequality, such as the $H^{\infty}$-well-posedness, can be adopted.

2) Though the definition of the well-posedness in [3] is not the same as ours, we can easily derive Theorem 4.1 and 7.1 in [3] from the proof of the above theorem. Especially, we can omit the assumption that $P$ satisfies Condition $\left(U_{\Gamma(x, \eta)}\right)$ and can relax the condition $p \geqq q$ to $p+1>q$ in Theorem 4.1 in [3].

3) Theorem 3.1 in [5] can be essentially restated as follows in our setting. (The statement of Theorem 3.1 in [5] is wrong (perhaps misprinted). The condition $(N)$ must be "for $|\alpha|+\langle\beta-\alpha, p\rangle\left\langle r\left(1-p_{0}\right)-(m-s)\right.$ ".)

Theorem. (Theorem 3.1 in [5]) For a rational $\kappa>0$, put $\Delta_{r, \kappa}=\{(j, \mu) ; r \leqq j$ $\leqq m, \mu \geqq 0\} \cup\{(j, \mu) ; 0 \leqq j \leqq r, \mu \geqq \kappa(r-j)\}$ and $\Delta_{r, \kappa}^{\sim}=\{(j, \mu) ; 0 \leqq j \leqq m, \mu \geqq \kappa(r-j)\}$ $(1 \leqq r \leqq m)$. Assume that $q_{0}+\kappa>q_{j}(j=1, \cdots, n), p_{m}^{(r, 0)}\left(0,0 ; 0, e_{n}\right) \neq 0$ and the following condition:

$$
\text { If }\left(j+|\alpha|, q_{0}(k+|\alpha|)+\langle q, \beta-\alpha\rangle\right) \notin \widetilde{\Delta_{r, k}} \text {, then } p_{m,(k, 3)}^{(j, \alpha)}\left(0,0 ; 0, e_{n}\right)=0 \text {. }
$$

If the Cauchy problem for $P$ is well-posed on $\Omega^{+}$, then the following holds.

$$
\begin{aligned}
& \text { If }\left(j+|\alpha|+h, q_{0}(k+|\alpha|+h)+\langle q, \beta-\alpha\rangle\right) \notin \Delta_{r, k}^{\sim} \text {, then } \\
& p_{m-h,(k, \beta)}^{(j, \alpha)}\left(0,0 ; 0, e_{n}\right)=0(h=1, \cdots, m) .
\end{aligned}
$$

Thus, our theorem is essentially an extension of Theorem 3.1 in [5].

Example 1.4. In the followings, let $n=1$ and assume that the Cauchy problem is well-posed in a neighborhood of $(0,0)$.

1) Let $m=3$ and $p_{3}=(\tau-x \xi)\left(\tau-x^{2} \xi\right) \tau$. Applying Theorem 1.2 with $q_{0}=\varepsilon$ (arbitrary positive number) and $q_{1}=1$, we get the condition that $p_{2}=a(t, x) \tau^{2}+$ $x b(t, x) \tau \xi+x^{3} c(t, x) \xi^{2}$ and $p_{1}=d(t, x) \tau+x e(t, x) \xi$ near $(0,0)$ for some $C^{\infty}$-functions $a, b, c, d, e$. This is also a sufficient condition for well-posedness. (See Example 2.7.)

2) Let $m=3$ and $p_{3}=(\tau-x \xi)\left(\tau-x^{2} \xi\right)\left\{\tau-\left(x+x^{2}\right) \xi\right\}$. Applying Theorem 1.2 with $q_{0}=\varepsilon$ (arbitrary positive number) and $q_{1}=1$, we get the condition that $p_{2}=a(t, x) \tau^{2}+x b(t, x) \tau \xi+x^{2} c(t, x) \xi^{2}$ and $p_{1}=d(t, x) \tau+x e(t, x) \xi$ near $(0,0)$ for some $C^{\infty}$-functions $a, b, c, d, e$. However, this is not sufficient for well-posedness. This condition does not reflect the contact-order of characteristic roots $x \xi$ and $\left(x+x^{2}\right) \xi$. If we apply Theorem 1.2 after transforming $P$ by the coordinate transformation $s=t, y=x e^{\tau}$, then we get a further condition $a(t, 0)+b(t, 0)+$ $c(t, 0)=1$. These conditions are sufficient for well-posedness. (The sufficiency follows from Theorem 2.2 in $\S 2$.)

3) Let $m=4$ and $p_{4}=\left\{\tau^{2}-\left(t^{2}+x^{2}\right) \xi^{2}\right\}\left\{\tau^{2}-\left(t^{2}+x^{2}\right)^{2} \xi^{2}\right\}$. Put $p_{3}=\sum_{i=0}^{3} a_{\imath}(t, x) \tau^{i} \xi^{3-i}$. Applying Theorem 1.2 with $q_{0}=q_{1}=1$, we get $a_{1}(0,0)=\left(\partial_{t}^{j} \partial_{x}^{k} a_{0}\right)(0,0)=0$ for $j+k \leqq 2$. The author does not know whether these conditions are sufficient or not. 


\section{$\S 2 . \quad$ A Necessary and Sufficient Condition to a Class of $t$-Involutive Operators}

In this section, put $\Omega^{+}=[0, T] \times \boldsymbol{R}^{n}$ and assume that the coefficients of $p_{m}$ are bounded on $\Omega^{+}$.

Definition 2.1. 1) We say that $p_{m}$ (or $P$ ) is t-involutive (resp. involutive) if the following two conditions are satisfied. (M. Zeman [18], K. Yamamoto [17].)

i) $p_{m}$ has the smooth real characteristic roots. That is, there exist $\lambda_{j}(t, x ; \xi) \in C^{\infty}\left(\Omega^{+} \times \dot{\boldsymbol{R}}^{n}\right)(j=1, \cdots, m)$ such that $\lambda_{j}$ are real-valued and $p_{m}(t, x ; \tau, \xi)$ $=\prod_{j=1}^{m}\left(\tau-\lambda_{j}(t, x ; \xi)\right)$.

ii) For any $1 \leqq j, k \leqq m$, there exists $A_{j, k}(t, x ; \xi) \in C^{\infty}\left(\Omega^{+} \times \dot{\mathbb{R}}^{n}\right)$ such that

$$
\begin{aligned}
\left\{\tau-\lambda_{j}(t, x ; \xi), \tau-\lambda_{k}(t, x ; \xi)\right\} & =\frac{1}{t} A_{j, k}(t, x ; \xi)\left(\lambda_{j}(t, x ; \xi)-\lambda_{k}(t, x ; \xi)\right) \\
(\operatorname{resp} . & \left.=A_{j, k}(t, x ; \xi)\left(\lambda_{j}(t, x ; \xi)-\lambda_{k}(t, x ; \xi)\right)\right),
\end{aligned}
$$

where $\{\cdot, \cdot\}$ is the Poisson bracket w. r.t. $(t, x ; \tau, \xi)$.

2) Put $\partial_{\jmath}=D_{t}-\lambda_{j}\left(t, x ; D_{x}\right)$. For $J \subset I=\{1, \cdots, m\}$, put $\pi_{J}=\prod_{\jmath \in J} \partial_{j}$, where the order of the product is arbitrarily fixed. Let $|J|$ denote the number of the elements of $J$.

A sufficient condition for well-posedness of the Cauchy problem to $t$-involutive operators is given by K. Yamamoto [17]. (Cf. M. Zeman [18], H. Uryu [15], H. Kumano-go [6].) Let $\$^{p}(V)$ denote the class of all classical pseudodifferential operators of order $p$ on an open subset $V$ of $\boldsymbol{R}^{n}$.

Theorem 2.2. Assume that $p_{m}$ is t-involutive and that for any $J \varsubsetneqq I$, there exists $A_{J}\left(t, x ; D_{x}\right) \in C^{\infty}\left([0, T] ; \$^{0}\left(\boldsymbol{R}^{n}\right)\right)$ such that

$$
P=\pi_{I}+\sum_{J \rightleftharpoons I} t^{\mid J_{\mid}-m} A_{J}\left(t, x ; D_{x}\right) \pi_{J} .
$$

Then, the Cauchy problem for $P$ is well-posed on $\Omega^{+}$.

Remark 2.3. 1) In [17], there is proved only the $H^{\infty}$-well-posedness under the assumption that all the coefficients belong to $\mathscr{B}^{\infty}\left(\Omega^{+}\right)=\left\{f \in C^{\infty}\left(\Omega^{+}\right) ; \partial_{t}^{j} \partial_{x}^{\alpha} f\right.$ is bounded on $\Omega^{+}$for any $\left.(j, \alpha)\right\}$. However, we can prove that there exists a finite propagation speed (, that is, there holds the condition $(U)$ with $\mu_{0}=1$ of Definition 1.1 in [9]). (See Appendix 2.) Hence, the $C^{\infty}$-well-posedness holds under our assumption.

2) The condition that $P$ can be written as (2-2) is not always necessary for well-posedness as is shown in the following example. 
Example 2.4. Consider $P=D_{t}^{2}-\left(x^{2}+y^{2}\right)^{2} D_{c}^{2}-a(x, y) D_{x}$ in $\mathbb{R}^{3}$. The above condition is $\frac{a(x, y)}{x^{2}+y^{2}} \in C^{\infty}\left(\boldsymbol{R}^{2}\right)$, though the Cauchy problem for $P$ is well-posed if and only if $a(0,0)=\partial_{x} a(0,0)=\partial_{y} a(0,0)=0$. (The "only if" part follows from Theorem 1.2. The "if" part follows from Theorem 1 in [13].)

The purpose of this section is to give an answer to the question when the condition that $P$ can be written as $(2-2)$ is necessary for well-posedness. We make the following assumption [B] on $p_{m}$.

For any $(\hat{t}, \hat{x}) \in \Omega^{+}$, there exist a neighborhood $U$ of $(\hat{t}, \hat{x})$, a non-negative integer $n_{1}, C^{\infty}$-functions $C_{l}(t, x) \quad\left(l=1, \cdots, n_{1}\right)$ on $U$ and non-negative integers $\kappa(l ; j, k)\left(l=0,1, \cdots, n_{1} ; 1 \leqq j, k \leqq m\right)$ such that the following three conditions are satisfied.

[B]

i ) $C_{l}(\hat{t}, \hat{x})=0\left(l=1, \cdots, n_{1}\right)$.

ii) $\left\{\left(\operatorname{grad}_{x} C_{l}\right)(\hat{t}, \hat{x}) ; l=1, \cdots, n_{1}\right\}$ are linearly independent.

iii) For any $1 \leqq j, k \leqq m$, there holds either of the followings.

a) $\lambda_{\jmath}(t, x ; \xi)-\lambda_{k}(t, x ; \xi)=t^{\kappa(0 ; \jmath, k)} \prod_{l=1}^{n_{1}} C_{l}(t, x)^{\kappa(l ; \jmath, k)} \Phi_{j, k}(t, x ; \xi)$, where $\Phi_{j, k} \in C^{\infty}\left(U \times \dot{R}^{n}\right)$ and $\Phi_{j, k}(t, x ; \xi) \neq 0$ on $U \times \dot{\mathbb{R}}^{n}$.

b) $\lambda_{\jmath}(t, x ; \xi)=\lambda_{k}(t, x ; \xi)$ on $U \times \dot{\mathbb{R}}^{n}$.

Theorem 2.5. Assume that $p_{m}$ is t-involutive and that $p_{m}$ satisfies the assumption [B]. The Cauchy problem for $P$ is well-posed on $\Omega^{+}$if and only if $P$ can be written as (2-2).

Remark 2.6. Assume that $\kappa(0 ; j, k)=0$ for any $j, k$. Then, $p_{m}$ is $t$-involutive if and only if $p_{m}$ is involutive. Further, in this case, $P$ can be written as (2-2) if and only if $P$ can be written as follows.

$$
P=\pi_{I}+\sum_{J \subsetneq I} A_{J}\left(t, x ; D_{x}\right) \pi_{J},
$$

where $A_{J}\left(t, x ; D_{x}\right) \in C^{\infty}\left([0, T] ; S^{0}\left(\boldsymbol{R}^{n}\right)\right)$.

In some cases, the condition that $P$ can be written as $(2-2)$ (or $\left.(2-2)^{\prime}\right)$ is reduced to a simpler condition. (Example 1.4, 1), 2).)

Example 2.7. (Cf. [14]) Let $\sigma(x) \in C^{\infty}\left(\mathbb{R}^{n}\right)$ satisfy $\operatorname{grad}_{x} \sigma(x) \neq 0$ if $\sigma(x)=0$. Let $r$ be an integer such that $0 \leqq r \leqq m$ and let $\kappa(1) \leqq \kappa(2) \leqq \cdots \leqq \kappa(m-r)$ be non-negative integers. Assume the following three conditions. 


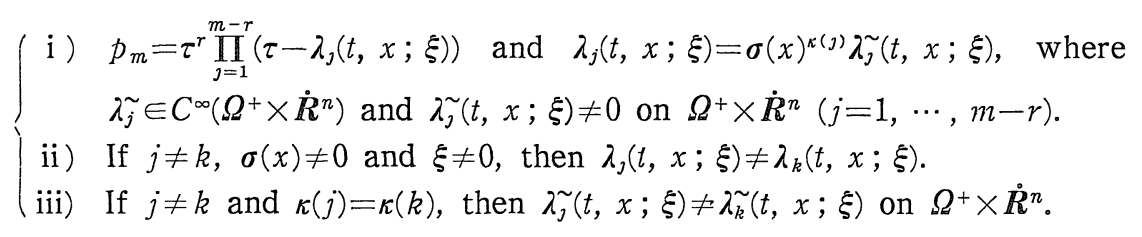

Let $P=\sum_{j+|\alpha| \leq m} a_{j, \alpha}(t, x) D_{t}^{j} D_{x}^{\alpha}\left(a_{j, \alpha} \in \mathscr{B}^{\infty}\left(\Omega^{+}\right)\right)$and put $\Gamma(j)=\sum_{l=1}^{m-j} \kappa(l)$ for $j=r, r+1$, $\cdots, m$. The Cauchy problem for $P$ is well-posed on $\Omega^{+}$if and only if there holds

$$
\left\{\begin{aligned}
& a_{j, \alpha}(t, x)= \sigma(x)^{\Gamma(m-|\alpha|)} b_{j, \alpha}(t, x) \\
& \text { for some } b_{j, \alpha} \in C^{\infty}\left(\Omega^{+}\right), \quad \text { if }|\alpha| \leqq m-r, \\
& a_{j, \alpha}(t, x)=0 \quad \text { on } \Omega^{+}, \quad \text { if }|\alpha|>m-r .
\end{aligned}\right.
$$

The "only if " part is easily proved by Theorem 1.2. The "if" part is proved by showing that $p_{m}$ is involutive and $P$ can be written as $(2-2)^{\prime}$. (Cf. $[16 ; \S 1]$.)

\section{$\S 3$. Proof of Theorem 1.2}

In this section, we shall prove Theorem 1.2. This theorem follows from the following proposition.

Proposition 3.1. Let $\kappa, \nu$ be positive rational numbers such that $q_{0}+\kappa>q_{j}$ $(1 \leqq j \leqq n)$. Assume the following three conditions.

1) $p_{m,(k, \beta)}^{(j, a)}\left(0,0 ; 0, e_{n}\right)=0$, if $q_{0}(k+|\alpha|)+\langle q, \beta-\alpha\rangle\langle\nu-\kappa(j+|\alpha|)$.

2) There exist $\hat{k}, \hat{\beta}, \hat{j}$ such that $p^{(\hat{j}, 0)},(\hat{k}, \hat{\beta})\left(0,0 ; 0, e_{n}\right) \neq 0$ and $q_{0} \hat{k}+\langle q, \hat{\beta}\rangle=\nu-\kappa \hat{j}$.

3) Either of the followings is satisfied.

Case (a) There holds $\nu=\kappa \hat{j}$ (, hence $\hat{k}=0, \hat{\beta}=0$ ).

Case $(b)$ There exist rational numbers $\kappa^{*}, \nu^{*}$ such that $0 \leqq \kappa^{*}<\kappa, \nu^{*}-\kappa^{*} \hat{j}$ $=\nu-\kappa \hat{j}$ and

$$
\begin{aligned}
& p_{m-h,(k, \beta)}^{(j, \alpha)}\left(0,0 ; 0, e_{n}\right)=0, \\
& \quad \text { if } \quad q_{0}(k+|\alpha|+h)+\langle q, \beta-\alpha\rangle\left\langle\nu^{*}-\kappa^{*}(j+|\alpha|+h) .\right.
\end{aligned}
$$

Under this assumption, if the Cauchy problem for $P$ is well-posed on $\Omega^{+}$, then there holds the following.

$$
\begin{aligned}
& p_{m-h,(k, \beta)}^{(j, \alpha)}\left(0,0 ; 0, e_{n}\right)=0, \\
& \quad \text { if } \quad q_{0}(k+|\alpha|+h)+\langle q, \beta-\alpha\rangle<\nu-\kappa(j+|\alpha|+h) .
\end{aligned}
$$

Proof of Theorem 1.2 via Proposition 3.1. If $\Delta$ has a form of type (A), the assumption of Proposition 3.1 with Case (a) in 3) holds for any $\kappa>0$ with $\hat{j}=m$, $\nu=\kappa m, \hat{k}=0, \hat{\beta}=0$. Hence, we get the desired result. Consider the case of type (B). If $\tilde{\kappa_{1}}=\kappa_{1}>0$, then put $\hat{j}=m$, and if $\kappa_{1}=0$, then put $\hat{j}=j_{1}$. Let $\nu_{1}^{\tilde{1}}=\tilde{\kappa_{1}} \tilde{j}$. In the case that $\kappa_{1}>0$, put $\kappa=\tilde{\kappa_{1}}$ and $\nu^{2}=\tilde{\nu_{1}}$. Then, we can apply Proposition 
3.1 with Case (a) in 3) and we get (3-2). In the case that $\kappa_{1}^{\sim}=0$, put $\kappa=\varepsilon$ and $\nu=\varepsilon \hat{j}$ for an arbitrary positive number $\varepsilon$. We can also apply Proposition 3.1 and we get (3-2) for any $\kappa>0$ and $\nu=\kappa \hat{j}$. Hence, we get (3-2) for $\kappa=\nu=0$. Thus, we get (3-2) for $\kappa=\kappa_{1}^{\tilde{1}}, \nu=\boldsymbol{\nu}_{1}$. Next, we shall prove (3-2) for $\kappa=\kappa_{i}$, $\nu=\nu_{i}$ under the assumption that there holds (3-2) for $\kappa=\kappa_{i-1}, \nu=\nu_{i-1}$. (If $i=2$, then regard $\kappa_{1}$ as $\tilde{\kappa_{1}}$ and $\nu_{1}$ as $\tilde{\nu_{1}}$.) If $i=2$ and $\tilde{\kappa_{1}}=\kappa_{2}$, then we have nothing to prove. In the other cases, take $\hat{j}=j_{i-1}, \kappa=\kappa_{i}, \nu=\nu_{l}, \kappa^{*}=\kappa_{\imath-1}, \nu^{*}=\nu_{\imath-1}$. We can apply Proposition 3.1 with Case (b) in 3) and we get (3-2) for $\kappa=\kappa_{i}, \nu=\nu_{i}$. Thus, we have (3-2) for $\kappa=\kappa_{i}, \nu=\nu_{\imath}(i=2, \cdots, r)$. The conclusion of Theorem 1.2 is just $(3-2)$ for $\kappa=\tilde{\kappa_{1}}, \nu=\nu_{1}^{\sim}$ and $\kappa=\kappa_{\imath}, \nu=\nu_{i}(i=2, \cdots, r)$.

In the following proof, we shall write $\rho^{a}=\exp _{\rho} a$, when $a$ is complicated.

Proof of Proposition 3.1. This proposition is proved by modifying the method used by Ivrii [4], [5]. Since, the Cauchy problem for $P$ is well-posed on $\Omega^{+}$, we have the following energy inequality.

Lemma 3.2. For any compact set $K \subset \Omega^{+}$, there exist constants $C, L$ such that the following holds. For any $(\hat{t}, \hat{x}) \in K$ and for any $u \in C_{0}^{\infty}(K)=\left\{v \in C_{0}^{\infty}\left(\mathbb{R}^{n+1}\right)\right.$; supp $v \subset K\}$, there holds.

$$
|u(\hat{t}, \hat{x})| \leqq C \sup _{p+|\hat{\beta}| \leqq L} \sup _{(t, x) \in K, t \leqq \hat{t}}\left|\left(\partial_{t}^{p} \partial_{x}^{3} P u\right)(t, x)\right| .
$$

We may assume that $\Omega^{+}$is star-shaped, that is, for any $(t, x) \in \Omega^{+}$and any $0 \leqq \lambda \leqq 1$, there holds $(\lambda t, \lambda x) \in \Omega^{+}$. For a positive $\lambda$, consider the coordinate transformation:

$$
\left\{\begin{array}{l}
s=t \exp _{\rho}\left(\lambda q_{0}\right) \\
y_{J}=x_{\jmath} \exp _{\rho}\left(\lambda q_{\jmath}\right) \quad(j=1, \cdots, n)
\end{array} \quad(\rho \geqq 1) .\right.
$$

By this coordinate transformation, the operator $P$ is transformed to another operator $P_{\rho}$. We have

$$
\begin{aligned}
p_{\rho, m-h}(s, y ; \sigma, \eta) & =\sum_{k, \beta, \jmath, a^{\prime}} K_{h}\left(k, \beta, j, \alpha^{\prime}\right) s^{k} y^{3} \sigma^{\jmath} \eta^{\prime a^{\prime}} \eta_{n}^{m-h-\jmath-\left|a^{\prime}\right|} \\
& \times \exp _{\rho}\left[-\lambda\left\{q_{0} k+\langle q, \beta\rangle-q_{0} j-\left\langle q^{\prime}, \alpha^{\prime}\right\rangle-q_{n}\left(n-h-j-\left|\alpha^{\prime}\right|\right)\right\}\right],
\end{aligned}
$$

where $K_{h}\left(k, \beta, j, \alpha^{\prime}\right)=\left(k ! \beta ! j ! \alpha^{\prime} !\right)^{-1} p_{m-h,(k, \beta)}^{\left(j, a^{\prime}, 0\right)}\left(0,0 ; 0, e_{n}\right) . \quad\left(\eta^{\prime}=\left(\eta_{1}, \cdots, \eta_{n-1}\right)\right.$, $q^{\prime}=\left(q_{1}, \cdots, q_{n-1}\right)$, etc. $)$ For a real number $\gamma$, a $C^{\infty}$-function $\varphi$ and a positive number $\delta<1$, set $E=\exp \left\{i\left(\gamma y_{n} \rho+\varphi \rho^{\delta}\right)\right\}$. These are determined later. We have

$$
\begin{aligned}
E^{-1} \circ P_{\rho} \circ E & =\sum_{h, k, \beta, \jmath, \alpha^{\prime}} K_{h}\left(k, \beta, j, \alpha^{\prime}\right) s^{k} y^{\beta} \\
\times & \quad \exp _{\rho}\left[-\lambda\left\{q_{0} k+\langle q, \beta\rangle-\left(q_{0}-q_{n}\right) j-\left\langle q^{\prime}-q_{n}, \alpha^{\prime}\right\rangle-q_{n}(m-h)\right\}\right. \\
& \left.\left.\quad+\delta\left(j+\left|\alpha^{\prime}\right|\right)+m-h-j-\left|\alpha^{\prime}\right|\right]\right] \\
& \times\left\{\left(\partial_{s} \varphi\right)^{\jmath}\left(\partial_{y^{\prime}} \varphi\right)^{a^{\prime}} \gamma^{m-h-\jmath-\mid a^{\prime}}+R_{\rho, h, k, \beta, \jmath, \alpha^{\prime}}\left(s, y ; D_{s}, D_{y}\right)\right\},
\end{aligned}
$$


where $R_{\rho}$ are differential operators of order $m$ whose coefficients tend to 0 in $C^{\infty}\left(\Omega^{+}\right)$as $\rho \rightarrow \infty$. Denote the exponent of $\rho$ by $d_{h}\left(k, \beta, j, \alpha^{\prime}\right)$. That is,

$$
\begin{aligned}
d_{h}\left(k, \beta, j, \alpha^{\prime}\right)= & \left(\lambda q_{0}-\lambda q_{n}+\delta-1\right) j+\left\langle\lambda q^{\prime}-\lambda q_{n}+\delta-1, \alpha^{\prime}\right\rangle \\
& -\lambda q_{0} k-\lambda\langle q, \beta\rangle+\left(\lambda q_{n}+1\right)(m-h) .
\end{aligned}
$$

Put $d_{\max }=d_{0}(\hat{k}, \hat{\beta}, \hat{j}, 0)=\left(\lambda q_{0}-\lambda q_{n}+\lambda \kappa+\delta-1\right) \hat{j}-\lambda \nu+\left(\lambda q_{n}+1\right) m$. Now, let $\varepsilon$ be the maximum of $\varepsilon^{\prime}$ that satisfies the following. (The maximum does exist if $\varepsilon=-\infty$ is admitted.)

There exist $h \geqq 1, k, \beta, j, \alpha^{\prime}$ such that

$$
\left(\begin{array}{l}
q_{0} k+\langle q, \beta\rangle+\left\langle q_{0}+\kappa-q^{\prime}, \alpha^{\prime}\right\rangle+\varepsilon^{\prime}\left(q_{0}+\kappa\right) h=\nu-\kappa j \\
\text { and } K_{h}\left(k, \beta, j, \alpha^{\prime}\right) \neq 0 .
\end{array}\right.
$$

If $\varepsilon \leqq 1$, then the conclusion of Proposition 3.1 holds.

Assume that $\varepsilon>1$. Let $\mathscr{M}=\left\{\left(h, k, \beta, j, \alpha^{\prime}\right) ; q_{0} k+\langle q, \beta\rangle+\left\langle q_{0}+\kappa-q^{\prime}, \alpha^{\prime}\right\rangle+\right.$ $\varepsilon\left(q_{0}+\kappa\right) h=\nu-\kappa j$ and $\left.K_{h}\left(k, \beta, j, \alpha^{\prime}\right) \neq 0\right\}$. Note that $(0, \hat{k}, \hat{\beta}, \hat{j}, 0) \in \mathscr{N}$ and that there exists $\left(h, k, \beta, j, \alpha^{\prime}\right) \in \mathscr{M}$ such that $h \geqq 1$. We have

$$
\begin{aligned}
& d_{\max }-d_{h}\left(k, \beta, j, \alpha^{\prime}\right) \\
& \left.=\lambda\left\{q_{0} k+\langle q, \beta\rangle+q_{0}+\kappa-q^{\prime}, \alpha^{\prime}\right\rangle+\varepsilon\left(q_{0}+\kappa\right) h-\nu+\kappa j\right\} \\
& \quad+\left(\hat{j}-j-\left|\alpha^{\prime}\right|\right)\left(\lambda q_{0}-\lambda q_{n}+\lambda \kappa+\delta-1\right)+\left(\lambda q_{n}+1-\lambda \varepsilon q_{0}-\lambda \varepsilon \kappa\right) h .
\end{aligned}
$$

Since $\varepsilon>1$ and $q_{0}+\kappa>q_{n}$, we can take $\lambda>0$ and $0<\delta<1$ such that $\lambda q_{0}-\lambda q_{n}+\lambda \kappa$ $+\delta-1=0$ and $\lambda q_{n}+1-\lambda \varepsilon\left(q_{0}+\kappa\right)=0$. We take such $\lambda$ and $\delta$. If $K_{h}\left(k, \beta, j, \alpha^{\prime}\right)$ $\neq 0$, then $d_{\max } \geqq d_{h}\left(k, \beta, j, \alpha^{\prime}\right)$ and the equality holds only if $\left(h, k, \beta, j, \alpha^{\prime}\right) \in \mathscr{M}$. we have

Put $\Phi\left(s, y, \gamma ; \sigma, \eta^{\prime}\right)=\sum_{\left(h, k, \beta, j, a^{\prime}\right) \in \mathcal{M}} K_{h}\left(k, \beta, j, \alpha^{\prime}\right) s^{k} y^{\beta} \sigma^{j} \eta^{\prime a^{\prime}} \gamma^{\hat{j}-\jmath-\left|\alpha^{\prime}\right|-h}$. Then,

$$
E^{-1} \circ P_{\rho} \circ E=\rho^{d \max } \gamma^{m-\hat{j}}\left\{\Phi\left(s, y, \gamma ; \partial_{s} \varphi, \partial_{y^{\prime}} \varphi\right)+R_{\rho}^{\sim}\left(s, y ; D_{s}, D_{y}\right)\right\},
$$

where $R_{\rho}^{\sim}$ is a differential operator of order $m$ whose coefficients tend to 0 in $C^{\infty}\left(\Omega^{+}\right)$as $\rho \rightarrow+\infty$.

Lemma 3.3. If $\left(h, k, \beta, j, \alpha^{\prime}\right) \in \mathscr{M}$, then there hold either (i) $h=0, j=\hat{j}$ or (ii) $h+j<\hat{j}$.

Proof. Case (a). If $\nu=\kappa \hat{j}$, then

$$
\begin{aligned}
0 & =q_{0} k+\langle q, \beta\rangle+\left\langle q_{0}+\kappa-q^{\prime}, \alpha^{\prime}\right\rangle+\varepsilon\left(q_{0}+\kappa\right) h-\nu+\kappa j \\
& =q_{0} k+\langle q, \beta\rangle+\left\langle q_{0}+\kappa-q^{\prime}, \alpha^{\prime}\right\rangle+\kappa(j+h-\hat{j})+\left(\varepsilon q_{0}+\varepsilon \kappa-\kappa\right) h \\
& \geqq \kappa(j+h-\hat{j}) .
\end{aligned}
$$

Hence, there holds $h+j \leqq j$ and the equality holds only if $k=0, \beta=0, \alpha^{\prime}=0$, $h=0, j=\hat{j}$. 
Case (b). We have

$$
\begin{aligned}
\nu-\kappa j= & q_{0} k+\langle q, \beta\rangle+\left\langle q_{0}+\kappa^{*}-q^{\prime}, \alpha^{\prime}\right\rangle+\left(q_{0}+\kappa^{*}\right) h+\left(\kappa-\kappa^{*}\right)\left|\alpha^{\prime}\right| \\
& +\left\{(\varepsilon-1) q_{0}+\varepsilon \kappa-\kappa^{*}\right\} h \geqq \nu^{*}-\kappa^{*} j+\left(\kappa-\kappa^{*}\right)\left|\alpha^{\prime}\right| \\
& +\left\{(\varepsilon-1) q_{0}+\varepsilon \kappa-\kappa^{*}\right\} h .
\end{aligned}
$$

Since $\nu-\kappa \hat{j}=\nu^{*}-\kappa^{*} \hat{j}$ and $\kappa>\kappa^{*} \geqq 0$, we have $\left(\kappa-\kappa^{*}\right)(\hat{j}-j-h) \geqq\left(\kappa-\kappa^{*}\right)\left|\alpha^{\prime}\right|+$ $(\varepsilon-1)\left(q_{0}+\kappa\right) h \geqq 0$. Hence, we have $j+h \leqq \hat{j}$ and the equality holds only if $\alpha^{\prime}=0, \quad h=0, \quad j=\hat{j}$.

Lemma 3.4. (Cf. [5; Lemma 1.2]) There exist $(\hat{s}, \hat{y}) \in \Omega^{+}, \hat{\eta}^{\prime} \in \mathbb{R}^{n} \backslash\{0\}$, $\hat{\gamma} \in \boldsymbol{R}, \hat{\sigma} \in \boldsymbol{C}$ such that $\Phi\left(\hat{s}, \hat{y}, \hat{\gamma} ; \hat{\sigma}, \hat{\eta}^{\prime}\right)=0$ and $\operatorname{Im} \hat{\sigma}<0$.

Proof. Take $\theta=\max \left\{\frac{(\varepsilon-1)\left(q_{0}+\kappa\right) h}{\hat{j}-j} ;\left(h, k, \beta, j, \alpha^{\prime}\right) \in \mathscr{M}, j<\hat{j}\right\}(>0)$ and put $\mathscr{M}^{\prime}=\left\{\left(h, k, \beta, j, \alpha^{\prime}\right) \in \mathscr{M} ; \theta(\hat{j}-j)=(\varepsilon-1)\left(q_{0}+\kappa\right) h\right\}$. For a positive parameter $\omega$, we have

$$
\begin{aligned}
& \Phi\left(s \omega^{-q_{0}}, y \omega^{-q}, \gamma \omega^{\kappa+q_{0}} ; \sigma \omega^{q_{0}+0}, \eta^{\prime} \omega^{q^{\prime}}\right) \\
& =\exp _{\omega}\left\{-\nu+\left(\kappa+q_{0}+\theta\right) \hat{j}\right\}\left\{\Phi_{1}\left(s, y, \gamma ; \sigma, \eta^{\prime}\right)+o(1)\right\} \quad(\omega \rightarrow \infty),
\end{aligned}
$$

where

$$
\Phi_{1}\left(s, y, \gamma ; \sigma, \eta^{\prime}\right)=\sum_{\mathscr{S} h^{\prime}} K_{h}\left(k, \beta, j, \alpha^{\prime}\right) s^{k} y^{\beta} \sigma^{j} \eta^{\prime a^{\prime}} \gamma^{\hat{\jmath}-j-1 a^{\prime} \mid-h}
$$

Consider

$$
\Phi_{1}\left(s, y, \gamma ; \sigma, \gamma \eta^{\prime}\right)=\sum_{j, h} A_{j, h}\left(s, y, \eta^{\prime}\right) \sigma^{j} \gamma^{\hat{j}-j-h} .
$$

There exist $\left(s^{\sim}, y^{\sim}\right) \in \Omega^{+}, \eta^{\prime \sim} \in \mathbb{R}^{n} \backslash\{0\}, j^{*}, h^{*}$ such that $A_{\hat{\jmath}, 0}\left(s^{\sim}, y^{\sim}, \eta^{\prime \sim}\right) \neq 0$, $A_{j *, h^{*}}\left(s^{\sim}, y^{\sim}, \eta^{\prime \sim}\right) \neq 0, j^{*}+h^{*}<\hat{j}, h^{*} \geqq 1$. The root of the equation $\Phi_{2}(\sigma, \gamma)=$ $\Phi_{1}\left(s^{\sim}, y^{\sim}, \gamma ; \sigma, \gamma \eta^{\prime \sim}\right)=0$ have the Peuiseux expansion with respect to $\gamma \in \mathbb{C}$. Let $\sigma=a \gamma^{\mu}$, then

$$
\Phi_{2}(\sigma, \gamma)=\sum_{j, h} A_{\jmath}\left(s^{\sim}, y^{\sim}, \eta^{\prime \sim}\right) \sigma^{j} \gamma^{j \mu+\hat{\jmath}-j-h}
$$

If we take $\mu=\max \left\{\frac{\hat{j}-j-h}{\hat{j}-j} ; A_{j, h}\left(s^{\sim}, y^{\sim}, \eta^{\prime \sim}\right) \neq 0, j<\hat{j}\right\}$, then $0<\mu<1$ and $\Phi_{2}(\sigma, \gamma)$ $=0$ has a root $\sigma=a \gamma^{\mu}\{1+o(1)\}$ as $|\gamma| \rightarrow \infty$ with $a \neq 0$. If we take a suitable branch of $\gamma^{\mu}$, then there exist $\gamma^{\sim} \in \mathbb{R}, \sigma^{\sim} \in C$ such that $\Phi_{2}\left(\sigma^{\sim}, \gamma^{\sim}\right)=0$ and $\operatorname{Im} \sigma^{\sim}$ $<0$. Taking $\hat{s}=s^{\sim} \omega^{-q_{0}}, \hat{y}=y^{\sim} \omega^{-q}, \hat{\gamma}=\gamma^{\sim} \omega^{\kappa+q_{0}}, \quad \hat{\eta}^{\prime}=\eta^{\prime \sim} \omega^{q^{\prime}}$ for sufficiently large $\omega \in \mathbb{R}$, we have the desired result.

There exists a neighborhood $V$ of $\left(\hat{s}, \hat{y}, \hat{\eta}^{\prime}\right)$ in $\Omega^{+} \times \dot{\mathbb{R}}^{n}$ such that $\Phi(s, y, \hat{\gamma}$; $\left.\sigma, \eta^{\prime}\right)=0$ has a root $\sigma$ such that $\operatorname{Im} \sigma<0$ for any $\left(s, y, \eta^{\prime}\right) \in V$. There exist a non-empty open set $V_{1} \subset V$, a positive number $\theta$ and a real-analytic function $F\left(s, y, \eta^{\prime}\right)$ on $V_{1}$ such that $\operatorname{Im} F\left(s, y, \eta^{\prime}\right)<-\theta$ on $V_{1}$ and $\sigma=F\left(s, y, \eta^{\prime}\right)$ is a root of $\Phi\left(s, y, \hat{\gamma} ; \sigma, \eta^{\prime}\right)=0$ with constant multiplicity $d(\geqq 1)$. Retake and fix a point $\left(\hat{s}, \hat{y}, \hat{\eta}^{\prime}\right)$ in $V_{1}$. There exist a neighborhood $W$ of $(\hat{s}, \hat{y})$ and a real 
analytic function $\varphi$ on $W$ such that

$$
\left\{\begin{array}{l}
\partial_{s} \varphi=F\left(s, y, \partial_{y^{\prime}} \varphi\right) \\
\varphi(\hat{s}, y)=\left\langle\hat{\eta}^{\prime}, y^{\prime}\right\rangle+i|y-\hat{y}|^{2}
\end{array} \quad \text { on } W .\right.
$$

Note that

$$
\left(\partial_{\sigma}^{l} \partial_{\eta^{\prime}}^{\nu} \Phi\right)\left(s, y, \hat{\gamma} ; \partial_{s} \varphi, \partial_{y^{\prime}} \varphi\right)\left\{\begin{array}{l}
=0 \text { on } W, \text { if } l+|\nu|<d \\
\neq 0 \text { on } W, \text { if } l=d, \nu=0
\end{array}\right.
$$

and

$$
\operatorname{Im} \varphi(s, y) \geqq|y-\hat{y}|^{2}+\theta(\hat{s}-s) \quad \text { for } \quad s \leqq \hat{s}, \quad(s, y) \in W .
$$

Taking such $\varphi$ as the function in $E$, we have from (3-5),

$$
E^{-1} \circ P_{\rho} \circ E=\rho^{d_{\max }} \hat{\gamma}^{m-\hat{\jmath}} R_{\rho}^{\sim}\left(s, y ; D_{s}, D_{y}\right) .
$$

We shall show that $R_{\rho}^{\sim}$ has a suitable structure.

Lemma 3.5. There exists a differential operator

such that

$$
Q_{0}=\sum_{l+|\nu| \leqq d} \sum_{\jmath \in J_{0}} a_{j, l, \nu}(s, y) \rho^{\jmath \delta^{\prime}} D_{s}^{\iota} D_{y}^{\nu}
$$

(i) $J_{0}$ is a set of a finite number of rational numbers,

(ii) $\delta^{\prime}$ is a rational number such that $0 \leqq \delta^{\prime}<\delta$,

(iii) $a_{j, l, \nu}$ are analytic functions on $W, a_{0, d, 0}(s, y) \neq 0$ on $W$ and $a_{\jmath, l, \nu}(s, y)=0$ on $W$ if $j+l+|\nu|>d$,

(iv) for any $N$, the operator $R_{\tilde{\rho}}=\hat{\gamma}^{m-\hat{\jmath}} \rho^{d \delta} R_{\rho}^{\sim}-Q_{0}$ can be written as

$$
R \widetilde{\widetilde{\rho}}=\sum_{h=1}^{N+1} \rho^{-\grave{o} h} R_{h}\left(s, y ; D_{s}, D_{y}, \rho\right),
$$

where $R_{h}$ is a differential operator of order $\leqq d+h$ whose coefficients are bounded in $C^{\infty}(W)$ as $\rho \rightarrow \infty$. Further, the coefficients of $R_{h}(h \leqq N)$ are analytic on $W$.

Proof. For an arbitrary function $w(s, y)$, we have by Leibniz rule,

$$
P_{\rho}(E \cdot w)=\sum_{l+|\nu| \leqq m} \frac{1}{l ! \nu !} D_{s}^{l} D_{y}^{\nu}(w)\left(\partial_{\sigma}^{l} \partial_{\eta}^{\nu} P_{\rho}\right)(E),
$$

where $\partial_{\sigma}^{l} \partial_{\eta}^{\nu} P_{o}$ is a differential operator with the symbol $\partial_{\sigma}^{l} \partial_{\eta}^{\nu} p_{\rho}(s, y ; \sigma, \eta)$. We have

$$
E^{-1}\left(\partial_{\sigma}^{l} \partial_{\eta}^{\nu} P_{\rho}\right)(E)=l ! \nu ! \rho^{d_{\max }(l+|\nu|) \delta} \hat{\gamma}^{m-\hat{j}}\left\{\left(\partial_{\sigma}^{l} \partial_{\eta}^{\nu} \Phi\right)\left(s, y, \hat{\gamma} ; \partial_{s} \varphi, \partial_{y} \varphi\right)+o(1)\right\},
$$

by the repeated use of Corollary A.4 in [2]. Thus, we have

$$
E^{-1} P_{\rho}(E \cdot w)=\rho^{d \max -d \delta} \sum_{l+|\nu| \leq m} \sum D_{s}^{l} D_{y}^{\nu}(w) a_{\tilde{l}, \nu, \omega}^{\tilde{n}}(s, y) \rho^{\omega} .
$$

Here, the second sum is taken for rational numbers $\omega$ such that $\omega \leqq(d-|\nu|-l) \delta$. 
By (3-7), if $l+|\nu|<d$ and $\omega=(d-|\nu|-l) \delta$, then $a_{\tilde{l}, \nu, \omega}=0$ on $W$. Further, there holds $a_{d, 0,0}^{\tilde{a}}(s, y) \neq 0$ on $W$. Let $\delta^{\prime}=\max \left[\left\{\frac{\omega}{d-|\nu|-l} ; d>|\nu|+l, a_{l, \nu, \omega} \neq 0\right\} \cup\{0\}\right]$. We have $0 \leqq \delta^{\prime}<\delta$ and we can write

$$
\begin{aligned}
& E^{-1} P_{\rho}(E \cdot w)
\end{aligned}
$$

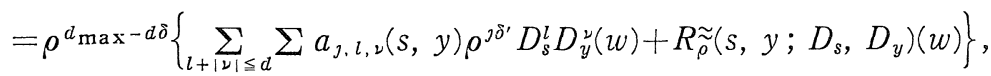

where the second sum is taken for $j \in J_{0}$ such that $j \leqq d-l-|\nu|$, and $J_{0}, a_{\jmath}, \nu$, $R \approx \widetilde{\rho}$ have the desired properties.

From now on, regard $(\hat{s}, \hat{y})$ as the origin $(0,0)$. Under this situation, from Lemma 3.2 , we have the following.

Proposition 3.6. For any compact set $K \subset W$, there exist positive constants $L, H, C$ such that there holds

$$
\begin{array}{r}
|u(0,0)| \leqq C \rho^{I I} \sup _{p+|\beta| \leq L} \sup _{\substack{s \leq 0 \\
(s, y) \in K}}\left|\left(\partial_{s}^{p} \partial_{y}^{\beta} P_{\rho} u\right)(s, y)\right| \\
\text { for any } u \in C_{0}^{\infty}(K) .
\end{array}
$$

We want to construct an asymptotic solution $u$ of $P_{\rho} u=0$ that violates the above inequality.

Definition 3.7. $([4])$ Let $d$ be a fixed positive integer. For a formal power series $g=g(\omega ; z)$ of $z=\left(z_{0}, z^{\prime}\right) \in C^{n+1}$ with coefficients depending on a parameter $\omega$, put

$$
\left(\begin{array}{l}
\Psi(g)(\omega ; \tau, \xi)=\sum_{a} \frac{1}{\alpha !} \tau^{\alpha_{0}} \xi^{\left|a^{\prime}\right|}\left|\left(\partial_{z}^{a} g\right)(\omega ; 0)\right|, \\
\Phi(g)(\omega ; \tau, \xi)=\sum_{k+\mid} \sum_{\beta \mid \leqq d-1} \sum_{a} \frac{1}{\alpha !} \tau^{a} \xi^{\left|a^{\prime}\right|}\left|\left(\partial_{z}^{a+\beta} g\right)(\omega ; 0)\right| \omega^{k},
\end{array}\right.
$$

which are formal power series of $(\tau, \xi)$.

The following is given in [4].

Proposition 3.8. Let $M$ be a positive integer, consider an operator $Q=$ $Q\left(\omega ; z, \partial_{z}\right)=\sum b_{a, j} \omega^{j} \partial_{z}^{\alpha}$ on a neighborhood $U$ of $0 \in \mathbb{C}^{n+1}$, where $b_{a, j}$ are holomorphic on $U, b_{(\alpha, 0, \cdots, 0), 0}=1$ and the sum is taken for a finite number of $(\alpha, j)$ such that $\alpha$ is a multi-index, $j$ is a rational number and $j+|\alpha| \leqq d$. There exist positive constants $K, \varepsilon_{1}$ and $\varepsilon_{2}$ depending only on $Q$ such that for any $a, b$ which satisfy $b \leqq \varepsilon_{1}$ and $0<a \leqq \varepsilon_{2} b$, there holds the following. If $v=v(\omega ; s, y)$ and $g=g(\omega ; s, y)$ satisfy 


$$
\left\{\begin{array}{l}
Q v=g, \\
\Psi(g) \ll\left(1-\frac{\tau}{a}-\frac{\xi}{b}\right)^{-n} \exp (K \omega \tau) . \\
\Phi(v)_{i \tau=0} \ll \omega^{-1}\left(1-\frac{\xi}{b}\right)^{-n}
\end{array}\right.
$$

then $v$ satisfies

$$
\Phi(v) \ll \omega^{-1}\left(1-\frac{\tau}{a}-\frac{\xi}{b}\right)^{-n} \exp (K \omega \tau),
$$

where $A \ll B$ means that $B$ is a majorant series of $A$.

Let $u(\omega ; s, y)$ be a formal power series of $(s, y)$ with coefficients depending on $\omega$. The following lemma is easily proved.

Lemma 3.9. (1) Consider an operator $R=\sum_{k \leqq H, \beta} a_{k, \beta}(\omega ; s, y) \partial_{s}^{k} \partial_{y}^{\beta}$, where $a_{k, \beta}(\omega ; s, y)=\sum_{l, \gamma} a_{k, \beta}^{(l, \gamma)}(\omega) s^{l} y^{\gamma} \ll \sum_{l, \gamma} A_{k, \beta}^{(l, \gamma)} s^{l} y^{\gamma}$ for any $\omega$ and $\sum_{l, \gamma} A_{k, \beta}^{(l, \gamma)} s^{l} y^{\gamma}$ is a convergent power series. If $\Psi(u) \ll\left(1-\frac{\tau}{a}-\frac{\xi}{b}\right)^{-n} \exp (K \omega \tau)$, then there exist positive constants $C, a^{\prime}$, $b^{\prime}$ such that $\Psi(R u) \ll C \omega^{H}\left(1-\frac{\tau}{a^{\prime}}-\frac{\xi}{b^{\prime}}\right)^{-n} \exp (K \omega \tau)$.

(2) If $\Psi(u) \ll A\left(1-\frac{\tau}{a}-\frac{\xi}{b}\right)^{-n} \exp (K \omega \tau)$, then for a sufficiently small neighborhood $V$ of $(0,0)$ and for any $(p, \beta)$, there exists a constant $C_{p, \beta}$ such that

$$
\left|\partial_{s}^{p} \partial_{y}^{\beta} u(\omega ; s, y)\right| \leqq C_{p, \beta} \omega^{p} \exp (K \omega|s|) \quad \text { on } \quad V \text {. }
$$

Now, we shall solve $\left(Q_{0}+R \widetilde{\tilde{\rho}}\right) u_{\rho}=0$ asymptotically. Consider $\rho^{\delta^{\prime}}$ as $\omega$. First, take $u_{0}(\rho ; s, y)$ such that $Q_{0} u_{0}=0$ and $\partial_{s}^{j} u_{01 s=0}=\delta_{0, j}$ for $0 \leqq j \leqq d-1\left(\delta_{0, j}\right.$ is the Kronecker's $\delta$ ). Since $\Phi\left(u_{0}\right)_{\mid \tau=0} \ll \rho^{(d-1) \delta^{\prime}}$, there holds

$$
\Phi\left(u_{0}\right) \ll\left(1-\frac{\tau}{a_{0}}-\frac{\xi}{b_{0}}\right)^{-n} \rho^{(d-1) \delta^{\prime}} \exp \left(K \rho^{\delta^{\prime}} \tau\right)
$$

for some positive constants $a_{0}, b_{0}, K$. Assume that $u_{0}, \cdots, u_{h}(0 \leqq h \leqq N-1)$ are taken such that

$$
\begin{gathered}
Q_{0} u_{\jmath}=-\sum_{l=1}^{j} \rho^{-l \delta} R_{l} u_{j-l}, \\
\Phi\left(u_{\jmath}\right) \ll C_{\jmath}\left(1-\frac{\tau}{a_{j}}-\frac{\xi}{b_{j}}\right)^{-n} \rho^{(d-1) \tilde{\delta}^{\prime}-\jmath\left(\hat{o}-\delta^{\prime}\right)} \exp \left(K \rho^{\delta^{\prime}} \tau\right), \\
\quad(j=0,1, \cdots, h),
\end{gathered}
$$

where $a_{0} \geqq a_{1} \geqq \cdots \geqq a_{h}>0$ and $b_{0} \geqq b_{1} \geqq \cdots \geqq b_{h}>0$. Then, there exist positive constants $A_{h, l}, a_{h+1}\left(\leqq a_{h}\right), b_{h+1}\left(\leqq b_{h}\right)$ such that $a_{h+1} \leqq \varepsilon_{2} b_{h+1}$ and 


$$
\Psi\left(R_{l} u_{h+1-\imath}\right) \ll A_{h, l}\left(1-\frac{\tau}{a_{h+1}}-\frac{\xi}{b_{h+1}}\right)^{-n} \rho^{(\iota+l) \hat{\delta}^{\prime}-(h+1-l)\left(\delta-\delta^{\prime}\right)} \exp \left(K \rho^{\delta^{\prime}} \tau\right) .
$$

Hence, there exists a constant $C_{h+1}$ such that

$$
\Psi\left(\sum_{l=1}^{h+1} \rho^{-l \hat{o}} R_{l} u_{h+1-l}\right) \ll C_{h+1}\left(1-\frac{\tau}{a_{h+1}}-\frac{\xi}{b_{h+1}}\right)^{-n} \rho^{(d+h+1) \hat{o}^{\prime}-(h+1) \delta} \exp \left(K \rho^{\hat{o}^{\prime}} \tau\right) .
$$

By Cauchy-Kowalevskaya theorem and Proposition 3.8, there exists $u_{h+1}$ such that

$$
\left\{\begin{array}{l}
Q_{0} u_{h+1}=-\sum_{i=1}^{h+1} \rho^{-l \grave{o}} R_{l} u_{h+1-l}, \\
\partial_{s}^{j} u_{h+1 \mid s=0}=0 \quad(j=0, \cdots, d-1), \\
\Phi\left(u_{h+1}\right) \ll C_{h+1}\left(1-\frac{\tau}{a_{h+1}}-\frac{\xi}{b_{h+1}}\right)^{-n} \rho^{(d+h) \hat{o}^{\prime}-(h+1) \grave{o}} \exp \left(K \rho^{\delta^{\prime}} \tau\right) .
\end{array}\right.
$$

Thus, we can take $u_{0}, \cdots, u_{N}$ such that

$$
\begin{aligned}
& \Psi\left(u_{\jmath}\right) \ll C_{\jmath}\left(1-\frac{\tau}{a_{j}}-\frac{\xi}{b_{j}}\right)^{-n} \rho^{-\jmath\left(\hat{o}-\hat{o}^{\prime}\right)} \exp \left(K \rho^{\hat{o}^{\prime}} \tau\right), \\
& E^{-1} P_{\rho}\left(E \sum_{j=0}^{N} u_{j}\right)=\rho^{d_{\max }-d \hat{o}}\left\{\sum_{h=1}^{N} \sum_{\jmath=N+1-h}^{N} \rho^{-h \grave{o}} R_{h} u_{j}+\rho^{-(N+1) \grave{o}} R_{N+1}\left(\sum_{\jmath=0}^{N} u_{j}\right)\right\} .
\end{aligned}
$$

For any $h=1, \cdots, N+1$ and any $(p, \beta)$, there exists a constant $C_{h, p, \beta}$ such that

$$
\left|\left(\partial_{s}^{p} \partial_{y}^{\beta} R_{h} u_{\jmath}\right)(s, y)\right| \leqq C_{h, p, \beta} \rho^{(d+h+p) \hat{o}^{\prime}-\jmath\left(\bar{\partial}-\hat{o}^{\prime}\right)} \exp \left(K \rho^{\delta^{\prime}}|s|\right)
$$

near $(0,0)$. Hence, there exist a neighborhood $W_{1}$ of $(0,0)$ and constants $C_{p, \beta}^{(N)}$ such that

$$
\begin{aligned}
\left|\partial_{s}^{p} \partial_{y}^{\beta} P_{\rho}\left(E \cdot \sum_{j=0}^{N} u_{j}\right)(s, y)\right| \leqq & C_{p, \beta}^{(N)} \rho^{d_{\max }+p+|\beta|-d \hat{o}+d \hat{o}^{\prime}-(N+1)\left(\hat{\delta}-\hat{o}^{\prime}\right)} \\
& \times \exp \left\{-\operatorname{Im} \varphi(s, y) \rho^{\hat{o}}+K \rho^{\hat{o}^{\prime}}|s|\right\} \quad \text { on } W_{1} .
\end{aligned}
$$

Since $-\operatorname{Im} \varphi(s, y) \leqq \theta s-|y-\hat{y}|^{2} \leqq-\theta|s|-|y-\hat{y}|^{2}$ for $s \leqq 0$, we have

$$
\left|\partial_{s}^{p} \partial_{y}^{\beta} P_{\rho}\left(E \cdot \sum_{j=0}^{N} u_{j}\right)(s, y)\right| \leqq C_{p, \beta}^{(N)} \rho^{d_{\max }+p+1,3 \mid-d \grave{o}+d \hat{o}^{\prime}-(N+1)\left(\delta-\delta^{\prime}\right)}
$$

on $W_{1} \cap\{s \leqq 0\}$ for sufficiently large $\rho$.

On the other hand, let $W_{2}$ be a relatively compact neighborhood of $(0,0)$ such that $\bar{W}_{2} \subset W_{1}$. There exists a positive number $\varepsilon$ such that for any $(p, \beta)$, there holds

$$
\left|\partial_{s}^{p} \partial_{y}^{\beta}\left(E \cdot \sum_{j=0}^{N} u_{j}\right)(s, y)\right| \leqq A_{p, \beta}^{(N)} \rho^{p+|\beta|} \exp \left(-\varepsilon \rho^{\delta}\right)
$$

on $\left(W_{1} \backslash W_{2}\right) \cap\{s \leqq 0\}$ for some constant $A_{p, \beta}^{(N)}$.

Now, we take $\chi(s, y) \in C_{0}^{\infty}\left(W_{1}\right)$ such that $\chi(s, y)=1$ on $W_{2}$. Then, by (3-13), 
(3-14), the asymptotic solution $u(s, y)=\chi(s, y) \cdot E \cdot \sum_{j=0}^{N} u$, violates the inequality (3-9) as $\rho \rightarrow+\infty$ for sufficiently large $N$.

\section{$\S 4$. Proof of Theorem 2.5}

In this section, assume that $p_{m}$ is $t$-involutive and satisfies the assumption [B]. Further, assume that the Cauchy problem for $P$ is well-posed on $\Omega^{+}$. Essentially, the proof of Theorem 2.5 goes along the same line as the proof of Main Theorem in [9]. For simplicity, we assume that $\lambda_{j} \not \equiv \lambda_{k}$ if $j \neq k$ and that $\kappa(0 ; j, k)=0$ for any $j, k$ (, hence $p_{m}$ is involutive). Fix an arbitrary $(\hat{t}, \hat{x}) \in \Omega^{+}$. We have only to show that there exists a neighborhood $V$ of $(\hat{t}, \hat{x})$ such that $P$ can be written as $(2-2)^{\prime}$ on $V$. The general case is proved by taking the arguments in [9] into account and modifying the following arguments.

Fix $1 \leqq \hat{l} \leqq n_{1}$ until Proposition 4.11. Since $\operatorname{grad}_{x} C_{\hat{\imath}}(\hat{t}, \hat{x}) \neq 0$, we can retake a coordinate on a neighborhood $U_{1}$ of $(\hat{t}, \hat{x})$ such that $C_{\hat{l}}(t, x)=x_{1}$.

Fix an arbitrary $z^{\sim}=\left(t^{\sim}, x^{\sim}\right) \in U_{1}$ such that $x_{1}^{\sim}=0$ and $C_{l}\left(t^{\sim}, x^{\sim}\right) \neq 0$ for all $l \neq \hat{l}$. Put $J[\hat{l}]=\{j \in\{1, \cdots, m\}$; there exists $k \neq j$ such that $\kappa(\hat{l} ; j, k) \neq 0\}$.

Lemma 4.1. If $j \in J[\hat{l}]$, then $\partial_{\xi_{1}} \lambda_{\jmath}(t, x ; \xi)$ is divisible by $x_{1}$.

Proof. There holds $\lambda_{j}(t, x ; \xi)-\lambda_{k}(t, x ; \xi)=x_{1}^{\kappa} \circ \phi(t, x ; \xi)$, where $\kappa=\kappa(\hat{l} ; j, k)$ $>0$ and $\phi$ is a $C^{\infty}$-function such that $\phi(t, x ; \xi) \neq 0$ if $C_{l}(t, x) \neq 0$ for all $l \neq \hat{l}$. Since

$$
\begin{aligned}
& \left\{\tau-\lambda_{\jmath}(t, x ; \xi), \tau-\lambda_{k}(t, x ; \xi)\right\}=\left\{\tau-\lambda_{j}, \lambda_{\jmath}-\lambda_{k}\right\} \\
& =x_{1}^{\kappa} \partial_{t} \phi-\sum_{i=1}^{n}\left(\partial_{\hat{\xi}_{i}} \lambda_{j}\right) x_{1}^{\kappa} \partial_{x_{\imath}} \phi-\left(\partial_{\xi_{1}} \lambda_{j}\right) \kappa x_{1}^{\kappa-1} \phi+\sum_{i=1}^{n}\left(\partial_{x_{\imath}} \lambda_{\jmath}\right) x_{1}^{\kappa} \partial_{\xi_{\imath}} \phi
\end{aligned}
$$

is divisible by $x_{1}^{\kappa}$, the function $\partial_{\xi_{1}} \lambda_{3}$ is divisible by $x_{1}$.

For $\hat{k} \in J[\hat{l}]$, the following holds.

Lemma 4.2. There exists a nowhere dense subset $S$ of $\boldsymbol{R}^{n}$ such that for any $\hat{\xi} \in \boldsymbol{R}^{n} \backslash S$, there exists a coordinate transformation $T=T\left(z^{\sim}, \hat{k}, \hat{\xi}\right)$ which satisfies the followings.

(1) $T$ transforms $z^{\sim}$ to $(0,0)$ and a neighbornood $W$ of $z^{\sim}$ onto a neighborhood $W^{\sim}$ of $(0,0)$.

(2) $T$ has a form $s=t-t^{\sim}, y=f(t, x)=\left(f_{1}(t, x), \cdots, f_{n}(t, x)\right)$. There hold $\operatorname{grad}_{x} f_{n \mid t=t^{\sim}}=\hat{\hat{\xi}}$ and that $f_{1}(t, x)=x_{1} g(t, x)$, where $g \in C^{\infty}(W)$ and $g(t, x) \neq 0$ on $W$.

(3) Put $\lambda_{\jmath}^{\sim}(s, y ; \eta)=\lambda_{j}\left(t, x ; \partial_{x}\langle f(t, x), \eta\rangle\right)-\partial_{t}\langle f(t, x), \eta\rangle$, where $\langle f(t, x), \eta\rangle$ $=\sum_{i} f_{i}(t, x) \eta_{i}$. There holds $\lambda \tilde{\hat{k}}\left(s, y ; e_{n}\right)=0$ on $W^{\sim}$.

Proof. We can take $h_{n}$ such that $\partial_{t} h_{n}=\lambda_{\hat{k}}\left(t, x ; \hat{\xi}+\partial_{x} h_{n}\right)$ and $h_{n \mid t=t \sim}=0$. Put $h_{\jmath}=0$ for $1 \leqq j \leqq n-1$. 
First, consider the case $n \geqq 2$. Put $S=\left\{\xi \in \mathbb{R}^{n} ; \xi_{j}=0\right.$, for $\left.2 \leqq j \leqq n\right\}$. If $\hat{\xi} \in \mathbb{R}^{n} \backslash S$, then we can take an invertible matrix $A$ such that ${ }^{t} A e_{n}=\hat{\xi}$ and $(A x)_{1}=x_{1}$. Putting $f(t, x)=A x+h(t, x)$, we get the desired result.

Next, consider the case $n=1$. Put $S=\{0\}$. If $\hat{\xi} \in \mathbb{R} \backslash\{0\}$, then put $f(t, x)$ $=\hat{\xi} x+h_{n}(t, x)$. Since $\partial_{t} f=\lambda_{\hat{k}}\left(t, x ; \partial_{x} f\right)=\partial_{x} f \cdot \partial_{\xi} \lambda_{\hat{k}}\left(t, x ; \partial_{x} f\right), f_{1 t=t \sim}=\hat{\xi} x$ and $\partial_{\xi} \lambda_{\hat{k}}(t, x ; \xi)$ is divisible by $x$, it is easy to see that $f(t, x)=x g(t, x)$ for a nonzero $C^{\infty}$-function $g$. Hence, we have the desired result.

Note that $p_{m}^{\tilde{m}}=\prod_{j=1}^{m}\left(\sigma-\lambda_{\jmath}\right)$ is the principal symbol of the operator $P^{\sim}$ which is transformed from $P$ by $T$. Put $C_{l}^{\sim}(s, y)=C_{l}(t, x)$. We may assume that $C_{l}^{\sim}(s, y) \neq 0$ on $W^{\sim}$ for all $l \neq \hat{l}$.

Fix $1 \leqq \hat{k} \leqq m$ and $\hat{\xi} \in \boldsymbol{R}^{n} \backslash S$. Denoting $\kappa(j)=\kappa(\hat{l} ; j, \hat{k})$, reorder $\{\kappa(j) ; j \neq \hat{k}\}$ as $\kappa\left(\rho_{1}\right) \leqq \cdots \leqq \kappa\left(\rho_{m-1}\right)$. Let $\Delta=\Delta\langle\hat{l} ; \hat{k}\rangle$ be the Newton polygon of size $m$ with the lower side $\Gamma(j)=\Gamma\langle\hat{l} ; \hat{k}\rangle(j)=\sum_{i=1}^{m-j} \kappa\left(\rho_{i}\right)(j=1, \cdots, m)$ and $\Gamma(0)=\infty$. (This is the Newton polygon determined by $\left(\kappa\left(\rho_{1}\right), \cdots, \kappa\left(\rho_{m-1}\right), \infty\right)$ in the terminology of $\S 3$ in [9].) For this Newton polygon, we get $\kappa_{1}, \cdots, \kappa_{r}$ as in Definition 0.3. Note that $\kappa_{j}$ are integers in this case. If $\kappa_{1}=0$, then modify $\Delta$ by setting $\tilde{\kappa_{1}}=1$ as in $\S 1$. Denote this Newton polygon by $\Delta^{\sim}=\Delta^{\sim}\langle\hat{l} ; \hat{k}\rangle$ and its lower side by $\mu=\Gamma^{\sim}(j)=\Gamma^{\sim}\langle\hat{l} ; \hat{k}\rangle(j)$. Now, put $q_{1}=1$ and $q_{j}=\varepsilon$ (arbitrary positive number) for $j \neq 1$. It is easy to see that $\Delta=\Delta\langle\hat{l} ; \hat{k}\rangle$ is the Newton polygon drawn from $P^{\sim}$ as in $\S 1$.

Lemma 4.3. The assumption [A] in $\S 1$ is satisfied for $P^{\sim}$ and $\Delta^{\sim}$.

Proof. The condition 1) of [A] is trivial. We have $p_{m}^{\sim}(s, y ; \sigma, \eta)=$ $\sum_{k=1}^{m}\left(\sigma-\lambda_{k}(s, y ; \eta)\right), \quad \lambda_{\hat{k}}\left(s, y ; e_{n}\right) \equiv 0, \quad \lambda_{k}\left(s, y ; e_{n}\right)=y_{1}^{\kappa(k)} \psi_{k}(s, y ; \eta)$. Further, by Lemma 4.1 , if $\kappa(k)>0$, then $\partial_{\eta_{1}} \lambda_{k}^{\sim}(s, y ; \eta)=y_{1} \phi_{k}(s, y ; \eta)$. (Here, $\phi_{k}, \phi_{k}$ are $C^{\infty}$-functions.) Hence, it is easy to see that $\partial_{\sigma}^{j} \partial_{\eta}^{\alpha} p_{m}^{\tilde{m}}\left(s, y ; 0, e_{n}\right)=y_{1}^{\Gamma^{\sim}(j+|a|)+\alpha_{1}}$ $\times \Phi_{j, \alpha}(s, y)$, where $\Phi_{j, a}$ is a $C^{\infty}$-function. (Here, regard $y_{1}^{\infty}$ as 0 .) Thus, if $q_{0}(k+|\alpha|)+\langle q, \beta-\alpha\rangle\left\langle\Gamma^{\sim}(j+|\alpha|)\right.$, then $\beta_{1}<\Gamma^{\sim}(j+|\alpha|)+\alpha_{1}$, hence $\partial_{s}^{k} \partial_{y}^{\beta} \partial_{\sigma}^{\jmath} \partial_{\eta}^{\alpha} p_{m}^{\sim}$ $\left(0,0 ; 0, e_{n}\right)=0$. This is the condition 2$)$.

Proposition 4.4. For $h=1, \cdots, m$, there holds $\operatorname{por}_{m-h,(k, \beta)}^{(j, a)}\left(0,0 ; 0, e_{n}\right)=0$ if $\beta_{1}<\Gamma^{\sim}(j+|\alpha|+h)+\alpha_{1}$.

Proof. By Theorem 1.2, we have $p_{m-\tilde{h},(k, \beta)}^{\left(j_{,}\right),}\left(0,0 ; 0, e_{n}\right)=0$ if $q_{0}(k+|\alpha|+h)$ $+\langle q, \beta-\alpha\rangle\left\langle\Gamma^{\sim}(j+|\alpha|+h)\right.$. Since $q_{0}(k+|\alpha|+h)+\langle q, \beta-\alpha\rangle=\beta_{1}-\alpha_{1}+\varepsilon(k+h+$ $\left.|\beta|-\beta_{1}+\alpha_{1}\right)$ and $\varepsilon>0$ is arbitrary, we have the desired result.

Now, we set up some classes of operators corresponding to a Newton polygon. Let $\Delta$ be a Newton polygon of size $m$ with the lower side $\Gamma(j)$ (see $\S 0)$. From now on, assume that all the Newton polygon that we treat satisfy 
$\Gamma(m-1)-\Gamma(m) \geqq 1$, that is, $\kappa_{1} \geqq 1$ in Definition 0.3. Further, we always extends $\Gamma(j)$ as $\Gamma(j)=\Gamma(m)-j+m$ for $j \geqq m$.

Definition 4.5. (Cf. $[9$; Definition 3.6]) (1) Let $p$ be an integer such that $0 \leqq p \leqq m$. We say $A=a\left(s, y ; D_{y}\right) \in \$^{p}\langle\Delta\rangle$ if $A$ satisfies the following conditions.

(i) $A \in C^{\infty}\left([0, \varepsilon) ; S^{p}(G)\right)$, where $\varepsilon$ is a positive number, $G$ is a neighborhood of $y=0$ and $[0, \varepsilon) \times G \subset W^{\sim}$.

(ii) Let $a(s, y ; \eta) \sim \sum_{l=0}^{\infty} a_{p-l}(s, y ; \eta)$ be the asymptotic expansion of the symbol $a$ of $A$, where $a_{p-l}$ is homogeneous of degree $p-l$ w.r.t. $\eta$. Then, there holds $a_{p-l,(k, \beta)}^{(\alpha)}\left(0,0 ; e_{n}\right)=0$ if $\beta_{1}<\Gamma(m-p+l+|\alpha|)+\alpha_{1}$.

(2) Let $H$ be an integer such that $0 \leqq H \leqq m$. We say $Q \in W_{H}\langle\Delta\rangle$ if $Q=\sum_{h=H}^{m} a_{h}\left(s, y ; D_{y}\right) D_{s}^{m-h}$, where $a_{h}\left(s, y ; D_{y}\right) \in \$^{h-H}\langle\Delta\rangle$.

Proposition 4.4 means that $P^{\sim} \in W_{0}\left\langle\Delta^{\sim}\langle\hat{l} ; \hat{k}\rangle\right\rangle$. We shall show some basic properties of the above classes of operators.

Definition 4.6. (1) For a positive integer $\kappa$, let $\Delta[\kappa]$ be the Newton polygon of size 1 with the lower side $\mu=\kappa-\kappa j(0 \leqq j \leqq 1)$. Let $\Delta[0]$ be the Newton polygon of size 1 with the lower side $\mu=-j(0 \leqq j \leqq 1)$. Put $\Delta[\infty]=\left\{(1, \mu) \in \mathbb{R}^{2} ; \mu \geqq 0\right\}$.

(2) Let $\Delta_{i}(i=1,2)$ be Newton polygons of size $m_{i}$ with the lower side $\Gamma_{i}(j)\left(0 \leqq j \leqq m_{i}\right)$. The Newton polygon $\Delta=\Delta_{1}+\Delta_{2}$ is the one of size $m_{1}+m_{2}$ with the lower side $\Gamma(j)=\min \left\{\Gamma_{1}(d)+\Gamma_{2}(e) ; d+e=j, \quad 0 \leqq d \leqq m_{1}, \quad 0 \leqq e \leqq m_{2}\right\}$ $\left(0 \leqq j \leqq m_{1}+m_{2}\right)$. (Cf. Definition 3.1 and Remark 3.2 in [9].)

Lemma 4.7. (1) Let $\lambda\left(s, y ; D_{y}\right) \in C^{\infty}\left([0, \varepsilon) ; S^{1}(G)\right)$ and let $\lambda_{0}(s, y ; \eta)$ be its principal symbol. Let $\kappa$ be a positive integer or $\infty$. If $\lambda_{0}\left(s, y ; e_{n}\right)=y_{1}^{\kappa} \phi(s, y)$ and $\partial_{\eta_{1}} \lambda_{0}(s, y ; \eta)=y_{1} \psi(s, y ; \eta)$ near $(0,0)$ for some $C^{\infty}$-function $\phi, \phi$, then $\lambda\left(s, y ; D_{y}\right) \in \$^{1}\langle\Delta[\kappa]\rangle$ and $D_{s}-\lambda\left(s, y ; D_{y}\right) \in W_{0}\langle\Delta[\kappa]\rangle$. Here, if $\kappa=\infty$, then regard $y_{1}^{\infty}$ as 0 .

(2) If $\lambda\left(s, y ; D_{y}\right) \in C^{\infty}\left([0, \varepsilon) ; S^{1}(G)\right)$, then $\lambda\left(s, y ; D_{y}\right) \in S^{1}\langle\Delta[0]\rangle$ and $D_{s}-$ $\lambda\left(s, y ; D_{y}\right) \in W_{0}\langle\Delta[0]\rangle$.

(3) Let $\Delta_{i}(i=1,2)$ be Newton polygons. If $A_{i} \in S^{p_{i}}\left\langle\Delta_{i}\right\rangle(i=1,2)$, then $A_{1} A_{2} \in S^{p_{1}+p_{2}}\left\langle\Delta_{1}+\Delta_{2}\right\rangle$. If $Q_{i} \in W_{H_{i}}\left\langle\Delta_{i}\right\rangle(i=1,2)$, then $Q_{1} Q_{2} \in W_{H_{1}+H_{2}}\left\langle\Delta_{1}+\Delta_{2}\right\rangle$.

Proof. (1) Assume $\kappa<\infty$. From the assumption, it is easy to see that $\lambda_{0,(k, \beta)}^{(a)}\left(0,0 ; e_{n}\right)=0$ if $\beta_{1}<\Gamma(|\alpha|)+\alpha_{1}$, where $\Gamma(j)=\kappa-\kappa j$ for $0 \leqq j \leqq 1$ and $\Gamma(j)=$ $1-j$ for $j \geqq 1$. Since $\Gamma(1+|\alpha|)+\alpha_{1} \leqq 0$ for any $\alpha$, we have the desired result. If $\kappa=\infty$, the proof is similar and easier.

(2) is obvious.

(3) Let $m_{i}$ be the size of $\Delta_{i}$ and $\Gamma_{i}(j)\left(0 \leqq j \leqq m_{i}\right)$ be the lower side of $\Delta_{i}$ $(i=1,2)$. It is easy to see that if we extend $\Gamma$ 's as already stated, then the 
lower side of $\Delta=\Delta_{1}+\Delta_{2}$ is $\Gamma(j)=\min \left\{\Gamma_{1}(d)+\Gamma_{2}(e) ; d+e=j, d \geqq 0, e \geqq 0\right\}$ for $j \geqq 0$. (Remember that we have assumed $\Gamma_{i}\left(m_{i}-1\right)-\Gamma_{\imath}\left(m_{\imath}\right) \geqq 1$.) Let $a_{i} \sim$ $\sum_{l=0}^{\infty} a_{i, p_{i}-l}$ be the asymptotic expansion of the symbol of $A_{i}(i=1,2)$. The asymptotic expansion of the symbol of $A_{1} A_{2}$ is $a \sim \sum_{L=0}^{\infty} a_{p_{1}+p_{2}-L}$, where

$$
a_{p_{1}+p_{2}-L}(s, y ; \eta)=\sum_{l+h+|\hat{\partial}|=L} \text { (const.) } a_{1, p_{1}-l}^{(\hat{)})}(s, y ; \eta) a_{2, p_{2}-h,(0, \hat{o})}(s, y ; \eta) .
$$

Hence,

$$
a_{p_{1}+p_{2}-L,(k, \beta)}^{(\alpha)}=\sum_{l+h+|\hat{o}|=L} \sum_{\gamma \leqq \alpha, p \leqq k, \omega \leqq \beta}(\text { const. }) a_{1, p_{1}-l,(p, \omega)}^{(\bar{o}+\gamma)} a_{2, p_{2}-h,(k-p, \delta+\beta-\omega)}^{(\alpha-\gamma)} .
$$

By the assumption, there holds $a_{1, p_{1}-l,(p, \omega)}^{(\hat{o}+\gamma)}\left(0,0 ; e_{n}\right)=0$ if $\omega_{1}<\Gamma_{1}\left(m_{1}-p_{1}+l+\right.$ $\mid \delta+\gamma i)+\delta_{1}+\gamma_{1}$, and there holds $a_{2, p_{2}-h,(k-p, \delta+\beta-\omega)}^{(\alpha-\gamma)}\left(0,0 ; e_{n}\right)=0$ if $\delta_{1}+\beta_{1}-\omega_{1}<$ $\Gamma_{2}\left(m_{2}-p_{2}+h+|\alpha-\gamma|\right)+\alpha_{1}-\gamma_{1}$. If $\omega_{1} \geqq \Gamma_{1}\left(m_{1}-p_{1}+l+|\delta+\gamma|\right)+\delta_{1}+\gamma_{1}$ and $\delta_{1}+\beta_{1}$ $-\omega_{1} \geqq \Gamma_{2}\left(m_{2}-p_{2}+h+|\alpha-\gamma|\right)+\alpha_{1}-\gamma_{1}$, then $\beta_{1} \geqq \Gamma\left(m_{1}+m_{2}-p_{1}-p_{2}+L+|\alpha|\right)+\alpha_{1}$. Hence, we get $a_{p_{1}+p_{2}-L,(k, \beta)}^{(\alpha)}\left(0,0 ; e_{n}\right)=0$ if $\beta_{1}<\Gamma\left(m_{1}+m_{2}-p_{1}-p_{2}+L+|\alpha|\right)+\alpha_{1}$. Thus, there holds $A_{1} A_{2} \in S^{p_{1}+p_{2}}\left\langle\Delta_{1}+\Delta_{2}\right\rangle$. Next, let $Q_{\imath}=\sum_{h=H_{i}}^{m_{i}} a_{h}^{(i)}\left(s, y ; D_{y}\right) D_{s}^{m_{i}-\hbar}$ $(i=1,2)$. We have $Q_{1} Q_{2}=\sum_{L=H_{1}+H_{2}}^{m_{1}+m_{2}} \sum_{h+k+l=L} a_{h}^{(1)}\left(s, y ; D_{y}\right)\left(D_{s}^{l} a_{k}^{(2)}\right)\left(s, y ; D_{y}\right) D_{s}^{m_{1}+m_{2}-L}$. Since $a_{h}^{(1)}\left(s, y ; D_{y}\right) \in S^{h-H_{1}}\left\langle\mathcal{A}_{1}\right\rangle$ and $\left(D_{s}^{l} a_{k}^{(2)}\right)\left(s, y ; D_{y}\right) \in S^{k-H_{2}}\left\langle\mathcal{A}_{2}\right\rangle$, we get $Q_{1} Q_{2}$ $\in W_{H_{1}+H_{2}}\left\langle\Delta_{1}+\Delta_{2}\right\rangle$.

So far, we have fixed $z^{\sim}, \hat{k}, \hat{\xi}$. We shall unite the above results for every $z^{\sim}, \hat{k}, \hat{\xi}$.

Definition 4.8. Let $H$ be an integer such that $0 \leqq H \leqq m$. We say $Q \in W_{H}(\hat{l})$ if the following conditions are satisfied.

(i) $Q=\sum_{h=H}^{m} a_{h}\left(t, x ; D_{x}\right) D_{t}^{m-h}$, where $a_{h}\left(t, x ; D_{x}\right) \in C^{\infty}\left([0, T] ; S^{h-I I}\left(R^{n}\right)\right)$.

(ii) For any $z^{\sim}=\left(t^{\sim}, x^{\sim}\right) \in U_{1}$ such that $x_{1}^{\sim}=0$ and $C_{l}\left(t^{\sim}, x^{\sim}\right) \neq 0$ for $l \neq \hat{l}$, any $\hat{k} \in J[\hat{l}]$ and any $\hat{\xi} \in \mathbb{R}^{n} \backslash S$, let $Q^{\sim}$ be the operator transformed from $Q$ by $T\left(z^{\sim}, \hat{k}, \hat{\xi}\right)$. Then, $Q^{\sim} \in W_{H}\left\langle\Delta^{\sim}\langle\hat{l}, \hat{k}\rangle\right\rangle$.

By Proposition 4.4, we have the following theorem.

Theorem 4.9. There holds $P \in W_{0}(\hat{l})$.

On the other hand, we have the following lemma.

Lemma 4.10. Let $A \in C^{\infty}\left([0, T] ; S^{0}\left(\boldsymbol{R}^{n}\right)\right)$ and $J \subset I$. Then, there holds $A \pi_{J} \in W_{m-|J|}(\hat{l})$.

Proof. Fix $z^{\sim}, \hat{k}, \hat{\xi}$, and let $\partial_{k}^{\sim}$ (resp. $A^{\sim}, \pi_{J}^{\tilde{J}}$ ) be the operator transformed from $\partial_{k}$ (resp. $A, \pi_{J}$ ) by $T\left(z^{\sim}, \hat{k}, \hat{\xi}\right)$. By Lemma 4.7 (1), (2), we have 
$\partial_{k}^{\sim} \in W_{0}\langle\Delta[\kappa(\hat{l} ; k, \hat{k})]\rangle$, where $\kappa(\hat{l} ; \hat{k}, \hat{k})=\infty$. Note that $1 \in W_{1}\langle\Delta[\kappa]\rangle$ for any $\kappa$ $(0 \leqq \kappa \leqq \infty)$, and $A^{\sim} \in W_{0}\left\langle\Delta_{0}\right\rangle$, where $\Delta_{0}=\{(0, \mu) ; \mu \geqq 0\}$. Since $\Delta^{\sim}\langle\hat{l} ; \hat{k}\rangle=$

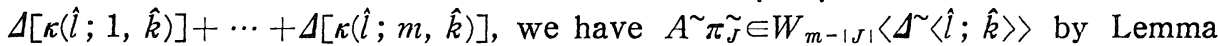
4.7 (3). Hence, there holds $A \pi_{J} \in W_{m-|J|}(\hat{l})$.

The following is the most important property of $W_{H}(\hat{l})$.

Proposition 4.11. Let $Q=\sum_{h=H}^{m} a_{h}\left(t, x ; D_{x}\right) D_{t}^{m-h} \in W_{H}(\hat{l}) \quad(H \geqq 1)$ and let its principal symbol be $q(t, x ; \tau, \xi)=\sum_{h=H}^{m} a_{h}^{0}(t, x ; \xi) \tau^{m-h}$, where $a_{h}^{0}$ is homogeneous of degree $h-H$ w.r.t. $\xi$. Then, for any $1 \leqq k \leqq m$ and $0 \leqq r \leqq m-H$, there holds

$$
\left(\partial_{\tau}^{r} q\right)\left(t, x ; \lambda_{k}(t, x ; \xi), \xi\right) \in x_{1}^{\Gamma\langle\hat{l} ; k\rangle(r+H)} \times C^{\infty}\left(V \times \dot{\boldsymbol{R}}^{n}\right),
$$

where $V$ is a neighborhood of $(\hat{t}, \hat{x})$.

In the original coordinate, there holds

$$
\left(\partial_{\because}^{r} q\right)\left(t, x ; \lambda_{k}(t, x ; \xi), \xi\right) \in C_{\hat{l}}(t, x)^{\Gamma<\hat{l}, k\rangle(r+H)} \times C^{\infty}\left(V \times \dot{\boldsymbol{R}}^{n}\right) .
$$

Proof. If $k \notin J[\hat{l}]$, then $\Gamma\langle\hat{l}, k\rangle(j)=0(1 \leqq j \leqq m)$, hence there is nothing to prove. Assume that $k \in J[\hat{l}]$. Fix $z^{\sim}, \hat{\xi}$ and let $Q^{\sim}$ be the operator transformed from $Q$ by $T\left(z^{\sim}, k, \hat{\xi}\right)$. Since $Q^{\sim} \in W_{H}\left\langle\Delta^{\sim}\langle\hat{l} ; k\rangle\right\rangle$, its principal symbol $q^{\sim}(s, y ; \sigma, \eta)$ satisfies that $\tilde{q}_{(0, \beta)}^{(r, 0)}\left(0,0 ; 0, e_{n}\right)=0$ if $\beta_{1}\left\langle\Gamma^{\sim}\langle\hat{l} ; k\rangle(r+H)\right.$. Since $q^{\sim}\left(0, y ; \sigma, e_{n}\right)=q\left(t^{\sim}, x ; \sigma+\lambda_{k}\left(t^{\sim}, x ; \hat{\xi}\right), \hat{\xi}\right)$ and $\Gamma\langle\hat{l} ; k\rangle(j)=\max \left(0, \Gamma^{\sim}\langle\hat{l} ; k\rangle(j)\right)$, we have $\partial_{x_{1}}^{j}\left\{\left(\partial_{\tau}^{r} q\right)\left(t^{\sim}, x ; \lambda_{k}\left(t^{\sim}, x ; \hat{\xi}\right), \hat{\xi}\right)\right\}_{\mid x=x^{\sim}}=0$ if $j<\Gamma\langle\hat{l} ; k\rangle(r+H)$. Since $\hat{\xi} \in \boldsymbol{R}^{n} \backslash S$ and $\left(t^{\sim}, x^{\sim}\right) \in U_{1}$ are arbitrary as long as $x_{1}^{\sim}=0$ and $C_{l}\left(t^{\sim}, x^{\sim}\right) \neq 0$ for $l \neq \hat{l}$, we have the desired result.

So far, we have fixed $\hat{l}$ arbitrarily. If $Q \in W_{\boldsymbol{H}}(l)$ for $l=1, \cdots, n_{1}(H \geqq 1)$, then for a sufficiently small neighborhood $V_{1}$ of $(\hat{t}, \hat{x})$ there holds

$$
\begin{array}{r}
\left\langle\partial_{\tau}^{r} q\right)\left(t, x ; \lambda_{k}(t, x ; \xi), \xi\right) \in \prod_{l=1}^{n_{1}} C_{l}(t, x)^{\Gamma\langle l ; k\rangle(r+H)} \times C^{\infty}\left(V_{1} \times \dot{\boldsymbol{R}}^{n}\right) \\
\text { for any } 1 \leqq k \leqq m, 0 \leqq r \leqq m-H,
\end{array}
$$

since $\left\{\operatorname{grad}_{x} C_{l}(t, x) ; l=1, \cdots, n_{1}\right\}$ is linearly independent.

Lemma 4.12. For any $1 \leqq \hat{k} \leqq m$, there exists an ordering $\left(\sigma_{1}, \cdots, \sigma_{m-1}\right)$ of $\{1, \cdots, m\} \backslash\{\hat{k}\}$ such that for any $1 \leqq l \leqq n_{1}$, there holds $\kappa\left(l ; \hat{k}, \sigma_{1}\right) \leqq \kappa\left(l ; \hat{k}, \sigma_{2}\right)$ $\leqq \cdots \leqq \kappa\left(l ; \hat{k}, \sigma_{m-1}\right)$.

Proof. Put $\rho(l ; j, k)=\min \{k(l ; \hat{k}, j), \kappa(l ; \hat{k}, k)\}$ for $j \neq \hat{k}, k \neq \hat{k}, j \neq k$. For such $j, k$, by the assumption [B]-iii)-a), there holds $\lambda_{3}(t, x ; \xi)-\lambda_{k}(t, x ; \xi)=$ $\left(\lambda_{\hat{k}}-\lambda_{k}\right)-\left(\lambda_{\hat{k}}-\lambda_{j}\right)=\left(\prod_{l=1}^{n_{1}} C_{l}(t, x)^{\rho(l ; j, k)}\right) \Psi_{j, k}(t, x ; \xi)$, where 


$$
\begin{aligned}
\Psi_{\jmath, k} & =\left(\prod_{l=1}^{n_{1}} C_{l}(t, x)^{\kappa(l ; \hat{k}, k)-\rho(l ; \jmath, k)}\right) \Phi_{\hat{k}, k}-\left(\prod_{l=1}^{n_{1}} C_{l}(t, x)^{\kappa(l ; \hat{k}, \jmath)-\rho(l ; \jmath, k)}\right) \Phi_{\hat{k}, j} \\
& =\left(\prod_{l=1}^{n_{1}} C_{l}(t, x)^{\kappa(l ; \jmath, k)-\rho(l ; \jmath, k)}\right) \Phi_{j, k} .
\end{aligned}
$$

Assume that there exist $l_{1}, l_{2}, j, k$ such that $\kappa\left(l_{1} ; \hat{k}, j\right)>\kappa\left(l_{1} ; \hat{k}, k\right)$ and $\kappa\left(l_{2} ; \hat{k}, k\right)$ $>\kappa\left(l_{2} ; \hat{k}, j\right)$. Note that $j, k, \hat{k}$ are distinct. By (4-3), neither $C_{l_{1}}$ nor $C_{l_{2}}$ divide $\Psi_{\jmath, k}$. Hence, by (4-4), if $C_{l}(t, x) \neq 0$ for $l \neq l_{1}, l_{2}$ and $\xi \neq 0$, then $\Psi_{j, k}(t, x ; \xi) \neq 0$. On the other hand, by (4-3), if $C_{l_{1}}(t, x)=C_{l_{2}}(t, x)=0$, then $\Psi_{j, k}(t, x ; \xi)=0$. These contradict each other, since there exists $(t, x)$ such that $C_{l}(t, x) \neq 0$ for $l \neq l_{1}, l_{2}$ and $C_{l_{1}}(t, x)=C_{l_{2}}(t, x)=0$. Thus, if $\kappa\left(l_{1} ; \hat{k}, j\right)>\kappa\left(l_{1} ; \hat{k}, k\right)$ for some $l_{1}$, then $\kappa(l ; \hat{k}, j) \geqq \kappa(l ; \hat{k}, k)$ for any $l$. The desired result easily follows from this.

Remark 4.13. The assumption ii) in [B] can be replaced by the following two conditions.

$($ ii) $-\alpha) \quad\left(\operatorname{grad}_{x} C_{l}\right)(\hat{t}, \hat{x})\left(l=1, \cdots, n_{1}\right)$ are pairwise linearly independent.

(ii) $\beta$ ) There holds the statement of Lemma 4.12 .

By using this lemma, the following proposition is proved similarly to Proposition 5.1 in [9].

Proposition 4.14. Let $q(t, x ; \tau, \xi)=\sum_{h=H}^{m} a_{h}(t, x ; \xi) \tau^{m-h}$ satisfy (4-2), where $a_{h} \in C^{\infty}\left(V_{1} \times \dot{\boldsymbol{R}}^{n}\right)$ is homogeneous of degree $h-H(H \geqq 1)$ w.r.t. $\xi$. Then, for any $J \subset I$ such that $|J|=m-H$, there exists $A_{J}(t, x ; \xi) \in C^{\infty}\left(V_{1} \times \dot{R}^{n}\right)$ such that $A_{J}$ is homogeneous of degree 0 w.r.t. $\xi$ and there holds

$$
q(t, x ; \tau, \xi)=\sum_{|J|=m-H} A_{J}(t, x ; \xi) \prod_{J \in J}\left(\tau-\lambda_{\jmath}(t, x ; \xi)\right) .
$$

Theorem 2.5 is proved similarly to $\S 6$ in [9], by combining Theorem 4.9, Lemma 4.10, Propositions 4.11 and 4.14 .

\section{Appendix 1. Proof of Remark 1.1, ii)}

Lemma A.1. Assume that $p_{m}(t, x ; \tau, \xi)$ is hyperbolic and $q_{j}(j=0, \cdots, n), \nu$ and $\kappa$ are positive rational numbers. If $\partial_{t}^{k} \partial_{x}^{\beta} \partial_{\tau}^{j} p_{m}\left(0,0 ; 0, e_{n}\right)=0$ for any $(k, \beta, j)$ such that $q_{0} k+\langle q, \beta\rangle\left\langle\nu-\kappa j\right.$, then $\partial_{t}^{k} \partial_{x}^{\beta} \partial_{\tau}^{j} \partial_{\xi}^{\alpha} p_{m}\left(0,0 ; 0, e_{n}\right)=0$ for any $(k, \beta, j, \alpha)$ such that $q_{0} k+\langle q, \beta\rangle\langle\nu-\kappa(j+|\alpha|)$.

Proof. For arbitrary $(\hat{t}, \hat{x})$, consider $f(t ; \tau, \xi)=p_{m}\left(\hat{t} t^{q_{0}}, \hat{x} t^{q} ; \tau, \xi\right)$. Then, $\partial_{\tau}^{\jmath} f\left(t ; 0, e_{n}\right) \in t^{\nu-\kappa \jmath} \times C^{0}[0, T]$. We can apply Proposition 3.2 in [8] when we regard $f(t ; \tau, \xi)$ as $P_{m}(t, \hat{x} ; \tau, \xi)$ and $\mu_{0}=1$. Thus, we get $\partial_{\tau}^{\jmath} \partial_{\xi}^{\alpha} f\left(t ; 0, e_{n}\right) \in$ $t^{\nu-\kappa(\jmath+|\alpha|)} \times C^{0}[0, T]$. Since $(\hat{t}, \hat{x})$ is arbitrary, this means that $\partial_{t}^{k} \partial_{x}^{\beta} \partial_{z}^{j} \partial_{\xi}^{\alpha} p_{m}(0,0$; $\left.0, e_{n}\right)=0$ for any $(k, \beta, j, \alpha)$ such that $q_{0} k+\langle q, \beta\rangle\langle\nu-\kappa(j+|\alpha|)$. 
If $q_{0} \geqq q_{j}(j=1, \cdots, n)$, then $q_{0}(k+|\alpha|)+\langle q, \beta-\alpha\rangle\langle\nu-\kappa(j+|\alpha|)$ implies $q_{0} k+\langle q, \beta\rangle\langle\nu-\kappa(j+|\alpha|)$. Hence, Remark 1.1, ii) follows from this lemma.

\section{Appendix 2. The Existence of a Finite Propagation Speed}

In this appendix, we shall give a rough sketch of the proof that there exists a finite propagation speed, as is stated in Remark 2.3, 1). First, assume that $p_{m}$ is involutive and has the form of $(2-2)^{\prime}$ on $\mathbb{R}^{n+1}$. We have only to show the invariance of the conditions under space-like coordinate transformations. Let $\varphi(x)$ be a $C^{\infty}$-function such that $\left|\operatorname{grad}_{x} \varphi\right|<\left[\sup \left\{\left|\lambda_{\jmath}(t, x ; \xi)\right| ;(t, x) \in \mathbb{R}^{n+1}\right.\right.$, $|\xi|=1, j=1, \cdots, m\}]^{-1}$. Consider a coordinate transformation $T ; s=t-\varphi(x)$, $y=x$. Let $P^{\approx}$ be the operator transformed from $P$ and put $P^{\sim}=\left\{p_{m}(s+\varphi(y)\right.$, $\left.\left.y ; 1,-\operatorname{grad}_{x} \varphi\right)\right\}^{-1} P \approx$. It is easy to see that $P^{\sim}$ is also involutive. The problem is whether $P^{\sim}$ can be written in the form of $(2-2)^{\prime}$. The main difficulty comes from the fact that $\lambda_{j}\left(t, x ; D_{x}\right)$ is not necessarily a pseudo-differential operator on $R^{n+1}$. In fact, $\lambda_{j}\left(t, x ; D_{x}\right)$ is pseudo-local on $\mathbb{R}^{n+1}$ if and only if $\lambda_{j}\left(t, x ; D_{x}\right)$ is a differential operator. (We say that an operator $Q ; \mathcal{E}^{\prime}\left(\mathbb{R}^{n+1}\right) \rightarrow \mathscr{D}^{\prime}\left(\mathbb{R}^{n+1}\right)$ is pseudo-local if singsupp $Q u \subset$ singsupp $u$ for any $u \in \mathcal{E}^{\prime}\left(\boldsymbol{R}^{n+1}\right)$.)

Let $S=\left\{(t, x ; \tau, \xi) \in \boldsymbol{R}^{n+1} \times \dot{\boldsymbol{R}}^{n+1} ; \tau=\lambda_{k}(t, x ; \xi)\right.$ for some $\left.k\right\}$. Take $\Delta_{j} \in S^{1}\left(\boldsymbol{R}^{n+1}\right)$ whose principal symbol is $\tau-\lambda_{\jmath}(t, x ; \xi)$ near $S$. Put $\Lambda_{J}=\prod_{j \in J} \Delta_{j}$ for $J \subset I$.

Proposition A.2. The operator $P$ can be written in the form of (2-2)' if and only if it can be written in the following form:

$$
P=\Lambda_{I}+\sum_{J \subsetneq I} B_{J} \Lambda_{J}+R,
$$

where $B_{J} \in S^{0}\left(\boldsymbol{R}^{n+1}\right), R \in S^{m}\left(\boldsymbol{R}^{n+1}\right)$ and the symbol of $R$ is rapidly decreasing near $S$.

The "only if" part of this proposition is almost trivial. The "if" part is proved by using the following lemma and noting that $P$ and $A_{J}\left(t, x ; D_{x}\right) \pi_{J}$ $(J \varsubsetneqq I)$ are differential operators w.r.t. $t$.

Lemma A.3. Let $\varphi_{l}(t, x ; \tau, \xi) \in C^{\infty}\left(\boldsymbol{R}^{n+1} \times \dot{\boldsymbol{R}}^{n+1}\right) \quad(l=1,2)$ be homogeneous functions of degree 0 w.r.t. $(\tau, \xi)$ such that $\varphi_{1}=1$ near $S, \varphi_{2}=1$ near $\operatorname{supp} \varphi_{1}$ and $\varphi_{2}=0$ near $\Gamma=\left\{(t, x ; \tau, \xi) \in \boldsymbol{R}^{n+1} \times \dot{\boldsymbol{R}}^{n+1} ; \xi=0\right\}$. Put $\Phi_{l}=\varphi_{l}\left(t, x ; D_{t}, D_{x}\right) \in$ $\$^{0}\left(\boldsymbol{R}^{n+1}\right)$. Assume that $B_{J}\left(t, x ; D_{t}, D_{x}\right) \in \mathbb{S}^{0}\left(\boldsymbol{R}^{n+1}\right)(|J|=m-h)$ and the principal symbol of $\sum_{|J|=m-h} B_{J}\left(t, x ; D_{t}, D_{x}\right) \pi_{J} \Phi_{2}$ is a polynomial of $\tau$ on $\operatorname{supp} \varphi_{1}$. Then, there exist $A_{J}\left(t, x ; D_{x}\right) \in C^{\infty}\left([0, T] ; S^{0}\left(\boldsymbol{R}^{n}\right)\right)(|J|=m-h)$ and $B_{K}\left(t, x ; D_{t}, D_{x}\right) \in$ $S^{0}\left(\boldsymbol{R}^{n+1}\right)(|K|<m-h)$ such that

$$
\begin{aligned}
& \sum_{|J|=m-h} B_{J}\left(t, x ; D_{t}, D_{x}\right) \pi_{J} \Phi_{1} \\
= & \sum_{|J|=m-h} A_{J}\left(t, x ; D_{x}\right) \pi_{J} \Phi_{1}+\sum_{|K|<m-h} B_{K}\left(t, x ; D_{t}, D_{x}\right) \pi_{K} \Phi_{1} .
\end{aligned}
$$


The latter condition in Proposition A.2 is clearly invariant under the coordinate transformation $T$. Thus, we have the desired result in the case of involutive operators on $\mathbb{R}^{n+1}$. The case of involutive operators on $\Omega^{+}=[0, T] \times \mathbb{R}^{n}$ is easily reduced to the case of $R^{n+1}$. The case of $t$-involutive operators is proved by the same arguments in the proof of Lemma 2.11 in [15]. (See also Theorem 3.3 in [6] or the last part of $\S 2$ in [9].)

\section{References}

[1] Chazarain, J., Opérateurs hyperboliques à caractéristiques de multiplicité constante, Ann. Inst. Fourier, Grenoble, 24 (1974), 173-202.

[2] Flaschka, H. and Strang, G., The correctness of the Cauchy problem, Advances in Math., 6 (1971), 347-379.

[3] Ivrii, V. Ya. and Petkov, V.M., Necessary conditions for the Cauchy problem for non-strictly hyperbolic equations to be well-posed, Russian Math. Surveys, 29 (1974), $1-70$.

[4] Ivrii, V. Ya., Conditions for correctness in Gevrey classes of the Cauchy problem for weakly hyperbolic equations, Sib. Math. J., 17 (1976), 422-435.

[5] - Cauchy problem conditions for hyperbolic operators with characteristics of variable multiplicity for Gevrey classes, Sib. Math. J., 17 (1977), 921-931.

[6] Kumano-go, H., Fundamental solution for a hyperbolic system with diagonal principal part, Comm. in Partial Differ. Equations, 4 (1979), 959-1015.

[7] Lax, A., On Cauchy's problem for partial differential equations with multiple characteristics, Comm. Pure Appl. Math., 9 (1956), 135-169.

[8] Mandai, T., Necessary conditions for well-posedness of the flat Cauchy problem and the regularity-loss of solutions, Publ. RIMS, Kyoto Univ., 19 (1983), 145-168.

[9] - A necessary and sufficient condition for the well-posedness of some weakly hyperbolic Cauchy problems, Comm. in Partial Differ. Equations, 8 (1983). 735-771.

[10] Mizohata, S., Some remarks on the Cauchy problem, J. Math. Kyoto Univ., 1 (1961), 109-127.

[11] Mizohata, S. and Ohya, Y., Sur la condition de E.E. Levi concernant des équations hyperboliques, Publ. RIMS, Kyoto Univ. Ser. A, 4 (1968), 511-526.

[12] - Sur la condition d'hyperbolicité pour les équations à caractéristiques multiples, II, Japan. J. Math., 40 (1971), 63-104.

[13] Olejnik, O. A., On the Cauchy problem for weakly hyperbolic equations, Comm. Pure Appl. Math., 23 (1970), 569-586.

[14] Sakamoto, R., Cauchy problem for degenerate hyperbolic equations, Comm. Pure Appl. Math., 33 (1980), 785-816.

[15] Uryu, H., The Cauchy problem for weakly hyperbolic equations, Comm. in Partial Differ. Equations, 5 (1980), 23-40.

[16] — The Cauchy problem for weakly hyperbolic equations (II) ; Infinite degenerate case, Tokyo J. Math., 3 (1980), 99-113.

[17] Yamamoto, K., The Cauchy problem for some class of hyperbolic differential operators with variable multiple characteristics, J. Math. Soc. Japan, 31 (1979), 481-502.

[18] Zeman. M., The well-posedness of the Cauchy problem for partial differential equations with multiple characteristics, Comm. in Partial Differ. Equations, 2 (1977), 223-249. 
